\title{
Local and Global Phaseless Sampling in Real Spline Spaces*
}

\author{
Wenchang Sun \\ School of Mathematical Sciences and LPMC, Nankai University, Tianjin 300071, China \\ Email: sunwch@nankai.edu.cn
}

\begin{abstract}
We study the recovery of functions in real spline spaces from unsigned sampled values. We consider two types of recovery. The one is to recover functions locally from finitely many unsigned samples. And the other is to recover functions on the whole line from infinitely many unsigned samples. In both cases, we give characterizations for a sequence of distinct points to be a phaseless sampling sequence, at which any nonseparable function is determined up to a sign on an interval or on the whole line by its unsigned sampled values. Moreover, for the case of local recovery, we also study the almost phaseless sampling and give a necessary and sufficient condition for a sequence of points to admit local recovery for almost all functions.
\end{abstract}

Keywords. Phase retrieval; phaseless sampling; spline functions; spline spaces.

MSC 2010: 42C15, 46C05.

\section{Introduction and Main Results}

The sampling theory is one of the most powerful results in signal analysis. It says that when a function satisfies certain conditions, it can be recovered from sampled values. In practice, it might happen that we have only intensity measurements. That is, sampled values are phaseless. To recover a function from intensity measurements, we have to study the problem of phase retrieval, which arises in the recovery of functions given the magnitude of its Fourier transform. We refer to the review paper [27] for an introduction on this topic.

Recently, many works have been done on the phase retrieval problem for general frames since Balan, Casazza and Edidin [4] introduced the concept of phaseless reconstruction in the setting of frame theory. For the case of finite-dimensional, various aspects to this problem which include the uniqueness and the stability of solutions were well studied [4 7, 10, 12, 15, 17, 20, 24, 25, 31]. Further generalizations including norm retrieval [3, 11] and phase retrieval from projections [9,15] were also studied.

*This work was partially supported by the National Natural Science Foundation of China (11371200, 11525104 and 11531013). 
For the case of infinite-dimensional, the problem becomes very different [1,2, 21-23, 28, 30,32. In particular, it was shown by Cahill, Casazza and Daubechies [8] that phase retrieval is never uniformly stable in the infinite-dimensional case. And in [13, 14, Chen, Cheng, Jiang, Sun and Wang studied phase retrieval of real-valued functions in shiftinvariant spaces. They gave some density results on the sequence of phaseless sampling points and studied the stability of phase retrieval. They showed that not all functions in such spaces can be recovered from intensity measurements. In particular, separable functions can not be recovered up to a sign. Recall that a function $f$ in a function space $H$ is said to be separable if $f=f_{1}+f_{2}$ for some $f_{1}, f_{2} \in H \backslash\{0\}$ with $f_{1}(x) f_{2}(x)=0$.

In this paper, we study the problem of phaseless sampling in real spline spaces. Specifically, let

$$
\varphi_{m}=\chi_{[0,1]} * \cdots * \chi_{[0,1]} \quad(m+1 \text { terms }), m \geq 1
$$

be the $m$-degree $\mathrm{B}$-spline and

$$
V_{m}=\left\{\sum_{k \in \mathbb{Z}} c_{n} \varphi_{m}(\cdot-n): c_{n} \in \mathbb{R}\right\}
$$

be the real spline space generated by $\varphi_{m}$. Note that $\varphi_{m}$ is compactly supported. The series is well defined on $\mathbb{R}$ for any real sequence $\left\{c_{n}: n \in \mathbb{R}\right\}$. The problem is to recover a function $f$ from its unsigned sampled values $\left|f\left(x_{i}\right)\right|$, where $\left\{x_{i}: i \in I\right\}$ is a sequence of sampling points.

One of the fundamental problems for the phaseless sampling is to determine sequences of sampling points which admit a local or global recovery of functions in given function spaces. In this paper, we study the characterization of sequences of sampling points at which we can recover any nonseparable function in spline spaces from unsigned samples.

We consider two types of phaseless sampling problems for functions in $V_{m}$. The one is local phaseless sampling, i.e., to recover functions on an finite interval from finitely many unsigned samples. Specifically, given two integers $N_{1}<N_{2}$ and a sequence of distinct points $E:=\left\{x_{i}: 1 \leq i \leq N\right\} \subset\left[N_{1}, N_{2}\right]$, we search for conditions on $E$ which guarantee a local reconstruction of $f$ on $\left[N_{1}, N_{2}\right]$ up to a sign from its unsigned sampled values $\left|f\left(x_{i}\right)\right|$, $1 \leq i \leq N$.

Local phaseless sampling is practically useful since measured signals are always time limited. For convenience, we introduce the following definition.

Let $\left.V_{m}\right|_{\left[N_{1}, N_{2}\right]}$ be the restriction of $V_{m}$ on $\left[N_{1}, N_{2}\right]$. That is, $\left.f \in V_{m}\right|_{\left[N_{1}, N_{2}\right]}$ if and only if $f=g \cdot \chi_{\left[N_{1}, N_{2}\right]}$ for some $g \in V_{m}$.

Definition 1.1 We call a sequence $E \subset\left[N_{1}, N_{2}\right]$ consisting of distinct points a (local) phaseless sampling sequence for $\left.V_{m}\right|_{\left[N_{1}, N_{2}\right]}$ if any nonseparable function $\left.f \in V_{m}\right|_{\left[N_{1}, N_{2}\right]}$ is determined up to a sign by its unsigned sampled values on $E$.

We give a necessary and sufficient condition for a sequence to be a local phaseless sampling sequence. 
Theorem 1.2 Let $N_{1}<N_{2}$ be integers. A sequence $E \subset\left[N_{1}, N_{2}\right]$ consisting of distinct points is a phaseless sampling sequence for $\left.V_{m}\right|_{\left[N_{1}, N_{2}\right]}$ if and only if it satisfies the followings,

$$
\begin{aligned}
\# E & \geq 2\left(N_{2}-N_{1}+m\right)-1 \\
\#\left(E \cap\left[N_{1}, N_{1}+k\right)\right) & \geq 2 k+m-1, \quad 1 \leq k \leq N_{2}-N_{1}, \\
\#\left(E \cap\left(N_{2}-k, N_{2}\right]\right) & \geq 2 k+m-1, \quad 1 \leq k \leq N_{2}-N_{1}, \\
\#\left(E \cap\left(n_{1}, n_{2}\right)\right) & \geq 2\left(n_{2}-n_{1}\right)-1, \quad N_{1} \leq n_{1}<n_{2} \leq N_{2},
\end{aligned}
$$

where \#E denotes the cardinality of a sequence $E$.

Moreover, if $E$ meets the above conditions and $\left|f_{1}(x)\right|=\left|f_{2}(x)\right|$ on $E$ for some $f_{1}, f_{2} \in$ $V_{m}$, then $\left|f_{1}(x)\right|=\left|f_{2}(x)\right|$ on $\left[N_{1}, N_{2}\right]$.

As shown in 13] 14, separable functions can not be recovered up to a sign from intensity measurements. Nevertheless, we see from Theorem 1.2 that if $E$ is a phaseless sampling sequence for $\left.V_{m}\right|_{\left[N_{1}, N_{2}\right]}$, then for any $\left.f \in V_{m}\right|_{\left[N_{1}, N_{2}\right]},|f(x)| \cdot \chi_{\left[N_{1}, N_{2}\right]}(x)$ is determined uniquely by its sampled values over $E$.

In [16], Fickus, Mixon, Nelson and Wang studied the problem of almost phase retrieval for general frames. Here "almost" means that for almost all functions in a finitedimensional function space, it is possible to recover the function from intensity measurements. When almost phase retrieval is considered, we need only very few measurements. In this paper, we study the problem of almost phaseless sampling on $\left.V_{m}\right|_{\left[N_{1}, N_{2}\right]}$. We give a characterization for a sequence to be an almost phaseless sampling sequence for $\left.V_{m}\right|_{\left[N_{1}, N_{2}\right]}$, which means that almost all functions in $\left.V_{m}\right|_{\left[N_{1}, N_{2}\right]}$ are uniquely determined up to a sign by their unsigned samples on such a sequence.

The other type of problem we are considered is the global phaseless sampling. That is, to recover a function on the whole line from its unsigned sampled values. Again, we study the construction of sequences $E \subset \mathbb{R}$ which admit a global phase retrieval, that is, any nonseparable function in $V_{m}$ is determined up to a sign by its unsigned sampled values on $E$. We call such sequences phaseless sampling sequences for $V_{m}$.

A characterization of phaseless sampling sequences for $V_{m}$ reads as followings.

Theorem 1.3 Let $E \subset \mathbb{R}$ be a sequence of distinct points. For $m \geq 2, E$ is a phaseless sampling sequence for $V_{m}$ if and only if it satisfies the following (P1) and (P2).

(P1) For any integers $n_{1}<n_{2}$, \# $\left(E \cap\left(n_{1}, n_{2}\right)\right) \geq 2\left(n_{2}-n_{1}\right)-1$;

(P2) For any integer $n_{0}$, there exist integers $n_{2}>n_{1} \geq n_{0}$ and $i_{1}<i_{2} \leq n_{0}$ such that $\#\left(E \cap\left[n_{1}, n_{2}\right]\right) \geq 2\left(n_{2}-n_{1}+m\right)-1$ and $\#\left(E \cap\left[i_{1}, i_{2}\right]\right) \geq 2\left(i_{2}-i_{1}+m\right)-1$.

For $m=1, E$ is a phaseless sampling sequence for $V_{1}$ if and only if it satisfies (P1) and

(P2') there exists an increasing sequence of integers $\left\{n_{k}: k_{1} \leq k \leq k_{2}\right\}$, which contains at least one point, such that $\#\left(E \cap\left[n_{k}-1, n_{k}\right]\right) \geq 3$ for $k_{1} \leq k \leq k_{2}$ and $\#(E \cap(n, n+$ $1))=2$ for $n \notin\left[n_{k_{1}}, n_{k_{2}}-1\right]$ if $k_{1}>-\infty$ or $k_{2}<\infty$. 
Moreover, if $\left|f_{1}(x)\right|=\left|f_{2}(x)\right|$ on $E$ for some $f_{1}, f_{2} \in V_{m}$ and $E$ meets (P1) and (P2) for $m \geq 2$ or (P1) and (P2') for $m=1$, then $\left|f_{1}(x)\right|=\left|f_{2}(x)\right|$ for any $x \in \mathbb{R}$.

Again, although separable functions can not be recovered up to a sign from intensity measurements, we see from Theorem 1.3 that if $E$ is a phaseless sampling sequence for $V_{m}$, then for any $f \in V_{m},|f(x)|$ is determined uniquely by its sampled values over $E$.

The paper is organized as follows. In Section 2, we study the problem of almost phaseless sampling in $\left.V_{m}\right|_{\left[N_{1}, N_{2}\right]}$ and give a necessary and sufficient condition for a sequence to be an almost phaseless sampling sequence. And in Sections 3 and 4, we give proofs of Theorem 1.2 and Theorem 1.3, respectively. In Section 5, we present some examples to illustrate the main results.

\section{Almost Phaseless Sampling in Spline Spaces}

In this section, we study the local recovery of almost all functions in spline spaces from phaseless sampled values.

Definition 2.1 We call $E=\left\{x_{i}: 1 \leq i \leq N\right\} \subset\left[N_{1}, N_{2}\right]$ an almost phaseless sampling sequence for $\left.V_{m}\right|_{\left[N_{1}, N_{2}\right]}$ if for any $\left.f \in V_{m}\right|_{\left[N_{1}, N_{2}\right]} \backslash H_{0}$, we can reconstruct $f$ up to a sign from the unsigned sample sequence $\left\{\left|f\left(x_{i}\right)\right|: 1 \leq i \leq N\right\}$, where $H_{0}$ consists of finitely many proper subspaces of $\left.V_{m}\right|_{\left[N_{1}, N_{2}\right]}$ and therefore is of Lebesgue measure zero.

The main result in this section is the following characterizations of almost phaseless sampling sequences.

Theorem 2.2 Let $E \subset\left[N_{1}, N_{2}\right]$ be a sequence of distinct numbers. Then $E$ is an almost phaseless sampling sequence for $\left.V_{m}\right|_{\left[N_{1}, N_{2}\right]}$ if and only if it satisfies the following conditions,

$$
\begin{aligned}
& \# E \geq N_{2}-N_{1}+m+1, \\
& \#\left(E \cap\left[N_{1}, N_{1}+k\right)\right) \geq k+1, \quad 1 \leq k \leq N_{2}-N_{1}, \\
& \#\left(E \cap\left(N_{2}-k, N_{2}\right]\right) \geq k+1, \quad 1 \leq k \leq N_{2}-N_{1}, \\
& \#\left(E \cap\left(n_{1}, n_{2}\right)\right) \geq n_{2}-n_{1}-m+1, \quad N_{1} \leq n_{1}<n_{2} \leq N_{2} .
\end{aligned}
$$

Note that the set consisting of all separable functions in $\left.V_{m}\right|_{\left[N_{1}, N_{2}\right]}$ is of $\left(N_{2}-N_{1}+m\right)$ dimensional Lebesgure measure zero (see Lemma 3.1). One might ask if it is possible to recover all nonseparable functions with an almost phaseless sampling sequence? The answer is unfortunately negative. In fact, we see from the characterization for local phaseless sampling sequences (Theorem 1.2) that if $E$ is only an almost phaseless sampling sequence, then many nonseparable functions are unrecoverable from its unsigned sampled values.

Before giving a proof of Theorem 2.2, we introduce some results on the almost phase retrieval for general frames.

We call a frame $\left\{f_{i}: 1 \leq i \leq N\right\}$ for $\mathbb{R}^{n}$ almost phase retrievable if for any $f \in \mathbb{R}^{n} \backslash E_{0}$, we can reconstruct $f$ up to a sign from the sequence of unsigned frame coefficients $\left\{\left|\left\langle f, f_{i}\right\rangle\right|\right.$ : 
$1 \leq i \leq N\}$, where $E_{0}$ consists of finitely many proper subspaces of $\mathbb{R}^{n}$ and therefore is of Lebesgue measure zero.

In [16, 33, some necessary and sufficient conditions for a frame to be almost phase retrievable were given. Here we give some further characterizations for almost phase retrievable frames.

Denote by $S_{N}=\left\{\left(s_{1}, \ldots, s_{N}\right): s_{1}=1, s_{i}= \pm 1, i \geq 2\right\}$. For an $n \times N$ matrix $A, A^{*}$ is the transpose of $A$ and $\mathcal{N}(A)$ is the null space of $A$, i.e., $\mathcal{N}(A)=\left\{x \in \mathbb{R}^{N}: A x=0\right\}$.

Since frames for $\mathbb{R}^{n}$ are equivalent to $n \times N$ matrices with rank $n$, for convenience, we also say a matrix is almost phase retrievable if its column vectors form an almost phase retrievable frame.

Let $\left.\varphi_{m}(\cdot-n)\right|_{\left[N_{1}, N_{2}\right]}$ be the restriction of $\varphi_{m}(\cdot-n)$ on $\left[N_{1}, N_{2}\right]$. That is,

$$
\left.\varphi_{m}(x-n)\right|_{\left[N_{1}, N_{2}\right]}= \begin{cases}\varphi_{m}(x-n), & x \in\left[N_{1}, N_{2}\right] \\ 0, & \text { otherwise }\end{cases}
$$

It is easy to see that $\left\{\left.\varphi_{m}(\cdot-n)\right|_{\left[N_{1}, N_{2}\right]}: N_{1}-m \leq n \leq N_{2}-1\right\}$ is a basis for $\left.V_{m}\right|_{\left[N_{1}, N_{2}\right]}$ [29, Lemma 4]. Therefore, $\left.\operatorname{dim} V_{m}\right|_{\left[N_{1}, N_{2}\right]}=N_{2}-N_{1}+m$.

Theorem 2.3 Suppose that $A$ is an $n \times N$ matrix whose column vectors form a frame for $\mathbb{R}^{n}, n \geq 2$. Let $s, s^{\prime} \in S_{N}$ and $s \neq s^{\prime}$. Then the followings are equivalent.

(i). A is almost phase retrievable.

(ii). $D_{s} A^{*} \mathbb{R}^{n} \neq D_{s^{\prime}} A^{*} \mathbb{R}^{n}$.

(iii). $\mathcal{N}\left(A D_{s}\right) \neq \mathcal{N}\left(A D_{s^{\prime}}\right)$.

(iv). $\operatorname{rank}\left(A D_{s}\right)<\operatorname{rank}\left(\begin{array}{c}A D_{s} \\ A D_{s^{\prime}}\end{array}\right)$.

(v). $\operatorname{rank}\left(M D_{s}\right)<\operatorname{rank}\left(\begin{array}{c}M D_{s} \\ M D_{s^{\prime}}\end{array}\right)$, where $M$ is an $(N-n) \times N$ matrix whose null space is the range of $A^{*}$.

Proof. Denote the column vectors of $A$ by $\varphi_{1}, \ldots, \varphi_{N}$.

(ii) $\Rightarrow$ (iii). Since $s \neq s^{\prime}$, there is some $i_{0} \geq 2$ such that $s_{i_{0}} \neq s_{i_{0}}^{\prime}$. Without loss of generality, we assume that $s_{i_{0}}=1$ and $s_{i_{0}}^{\prime}=-1$. Let $E=\left\{x \in \mathbb{R}^{n}:\left\langle x, \varphi_{1}\right\rangle=0\right.$ or $\left.\left\langle x, \varphi_{i_{0}}\right\rangle=0\right\}$. Then $E$ is of measure zero.

Assume that $D_{s} A^{*} \mathbb{R}^{n}=D_{s^{\prime}} A^{*} \mathbb{R}^{n}$. Then for any $x \in \mathbb{R}^{n}$, there is some $x^{\prime} \in \mathbb{R}^{n}$ such that $D_{s} A^{*} x=D_{s^{\prime}} A^{*} x^{\prime}$. Hence

$$
\left\langle x, \varphi_{1}\right\rangle=\left\langle x^{\prime}, \varphi_{1}\right\rangle \text { and }\left\langle x, \varphi_{i_{0}}\right\rangle=-\left\langle x^{\prime}, \varphi_{i_{0}}\right\rangle .
$$

It follows that for $x \notin E, x \neq \pm x^{\prime}$, which contradicts with (ii).

(iii) $\Rightarrow$ (ii). For $s, s^{\prime} \in S_{N}$ with $s \neq s^{\prime}$, define

$$
E_{s, s^{\prime}}=\left\{x \in \mathbb{R}^{n}: \text { there is some } x^{\prime} \in \mathbb{R}^{n} \text { such that } D_{s} A^{*} x=D_{s^{\prime}} A^{*} x^{\prime}\right\} .
$$


Since $D_{s} A^{*} \mathbb{R}^{n} \neq D_{s^{\prime}} A^{*} \mathbb{R}^{n}, D_{s} A^{*} \mathbb{R}^{n} \cap D_{s^{\prime}} A^{*} \mathbb{R}^{n}$ is a proper subspace of $D_{s} A^{*} \mathbb{R}^{n}$. Consequently, $E_{s, s^{\prime}}$ is a proper subspace of $\mathbb{R}^{n}$. Let

$$
E_{0}=\bigcup_{s, s^{\prime} \in S_{N}, s \neq s^{\prime}} E_{s, s^{\prime}}
$$

Then $E_{0}$ is the union of finitely many proper subspaces of $\mathbb{R}^{n}$. For any $x \in \mathbb{R}^{n} \backslash E_{0}$, if there is some $x^{\prime} \in \mathbb{R}^{n}$ such that $\left|\left\langle x, \varphi_{i}\right\rangle\right|=\left|\left\langle x^{\prime}, \varphi_{i}\right\rangle\right|$, then there is some $s \in S_{N}$ such that $A^{*} x=D_{s} A^{*} x^{\prime}$ or $A^{*} x=-D_{s} A^{*} x^{\prime}$. Hence $x=x^{\prime}$ or $x=-x^{\prime}$. In other words, $A$ is almost phase retrievable.

(iii) $\Leftrightarrow$ (iii). It follows from the fact that $\mathcal{N}\left(A D_{s}\right)=\left(D_{s} A^{*} \mathbb{R}^{n}\right)^{\perp}$.

(iii) $\Leftrightarrow$ (iv). Observe that $\operatorname{rank}\left(A D_{s}\right)=\operatorname{rank}\left(A D_{s^{\prime}}\right)$. If

$$
\operatorname{rank}\left(A D_{s}\right)=\operatorname{rank}\left(\begin{array}{c}
A D_{s} \\
A D_{s^{\prime}}
\end{array}\right)
$$

then there exists some invertible $n \times n$ matrix $P$ such that $A D_{s}=P A D_{s^{\prime}}$. And vice versa. It follows that (2.5) is equivalent to $\mathcal{N}\left(A D_{s}\right)=\mathcal{N}\left(A D_{s^{\prime}}\right)$. This proves the equivalence of (iii) and (iv).

$($ ii $) \Leftrightarrow(\mathbb{\nabla})$. See [33, Theorem 8].

Let $A$ be an $n \times N$ matrix with rank $n$. We say that $A$ is weak full spark if its rank remains unchanged when any one of its columns is removed.

For the case of $N=n+1$, we show that almost phase retrievable frames are equivalent to weak full spark matrices.

Theorem 2.4 Let the hypothesis be as in Theorem 2.3. Then we have

(i). If $A$ is almost phase retrievable, then $A$ is weak full spark.

(ii). Conversely, if $A$ is weak full spark and $N=n+1$, then $A$ is almost phase retrievable.

Proof. (i). Denote the column vectors of $A$ by $\varphi_{1}, \ldots, \varphi_{N}$. If $A$ is not weak full spark, then there is some $1 \leq i_{0} \leq N$ such that $\varphi_{i_{0}}$ can not be written as a linear combination of the others. Consequently, if

$$
\sum_{i=1}^{N} c_{i} \varphi_{i}=0,
$$

then we have $c_{i_{0}}=0$. Let $s=(1, \ldots, 1)$. If $i_{0}=1$, then set $s^{\prime}=(1,-1, \ldots,-1)$. Otherwise, set $s^{\prime}=(1, \ldots,-1, \ldots, 1)$ (only the $i_{0}$-th entry is -1$)$. In both cases, we have $s \neq s^{\prime}$ and $\mathcal{N}\left(A D_{s}\right)=\mathcal{N}\left(A D_{s^{\prime}}\right)$. By Theorem 2.3 (iii), $A$ is not almost phase retrievable, which contradicts with the hypothesis. Hence $A$ is weak full spark.

(ii). Assume that $A$ is not almost phase retrievable. By Theorem 2.3(iii), there exist some $s, s^{\prime} \in S_{N}$ with $s \neq s^{\prime}$ such that $\mathcal{N}\left(A D_{s}\right)=\mathcal{N}\left(A D_{s^{\prime}}\right)$.

Let $I_{1}=\left\{1 \leq i \leq N: s_{i}=s_{i}^{\prime}\right\}$ and $I_{2}$ be the complement of $I_{1}$. Let $c=\left(c_{1}, \ldots, c_{N}\right)^{*} \in$ $\mathbb{R}^{N}$ be such that

$$
A D_{s} c=0
$$


Then we have $A D_{s^{\prime}} c=0$. Hence

$$
\sum_{i \in I_{1}} c_{i} s_{i} \varphi_{i}=\sum_{i \in I_{2}} c_{i} s_{i} \varphi_{i}=0
$$

There are two cases.

(a) For $l=1$ or $l=2$, the solution of (2.6) satisfies that

$$
c_{i}=0, \quad i \in I_{l}
$$

In this case, for $i \in I_{l}, \varphi_{i}$ can not be written as a linear combination of other vectors which is impossible since $A$ is weak full spark.

(b) There is a solution of (2.6) such that neither $\left\{c_{i}: i \in I_{1}\right\}$ nor $\left\{c_{i}: i \in I_{2}\right\}$ is a sequence of zeros.

In this case, we see from (2.7) that

$$
\operatorname{rank}\left(\left\{\varphi_{i}: i \in I_{1}\right\}\right) \leq \# I_{1}-1 \text { and } \operatorname{rank}\left(\left\{\varphi_{i}: i \in I_{2}\right\}\right) \leq \# I_{2}-1 .
$$

Hence

$$
\operatorname{rank}\left(\left\{\varphi_{i}: 1 \leq i \leq N\right\}\right) \leq \# I_{1}+\# I_{2}-2=N-2=n-1,
$$

which is possible since $\left\{\varphi_{i}: 1 \leq i \leq N\right\}$ is a frame for $\mathbb{R}^{n}$.

Next we consider the reconstruction of functions in spline spaces from phaseless sampled values. We begin with some results on local sampling in spline spaces.

Definition 2.5 We call $E=\left\{x_{k}: 1 \leq k \leq K\right\}$ a (local) sampling sequence for $\left.V_{m}\right|_{\left[N_{1}, N_{2}\right]}$ if $E \subset\left[N_{1}, N_{2}\right]$ and there is a sequence of functions $\left\{S_{k}: 1 \leq k \leq K\right\}$ such that

$$
f(x)=\sum_{k=1}^{K} f\left(x_{k}\right) S_{k}(x), \quad \forall f \in V_{m}, x \in\left[N_{1}, N_{2}\right] .
$$

Based on the celebrated Schönberg-Whitney Theorem [26], the following characterization of local sampling sequences for spline spaces was proved in [29].

Proposition 2.6 A sequence $E \subset\left[N_{1}, N_{2}\right]$ of distinct points is a sampling sequence for $\left.V_{m}\right|_{\left[N_{1}, N_{2}\right]}$ if and only if it satisfies the following conditions,

$$
\begin{aligned}
& \# E \geq N_{2}-N_{1}+m, \\
& \#\left(E \cap\left[N_{1}, N_{1}+k\right)\right) \geq k, \quad 0 \leq k \leq N_{2}-N_{1}, \\
& \#\left(E \cap\left(N_{2}-k, N_{2}\right]\right) \geq k, \quad 0 \leq k \leq N_{2}-N_{1}, \\
& \#\left(E \cap\left(n_{1}, n_{2}\right)\right) \geq n_{2}-n_{1}-m, \quad N_{1} \leq n_{1}<n_{2} \leq N_{2} .
\end{aligned}
$$

Applying Theorem 2.3 to the local phaseless sampling in spline spaces, we get the following characterization of almost phaseless sampling sequences. 
Lemma 2.7 Let $E=\left\{x_{i}: 1 \leq i \leq N\right\} \subset\left[N_{1}, N_{2}\right]$ be a sequence of distinct numbers and $K:=N_{2}-N_{1}+m \leq N$. Define the $K \times N$ matrix $\Phi$ by

$$
\Phi=\left[\varphi_{m}\left(x_{j}-n\right)\right]_{N_{1}-m \leq n \leq N_{2}-1,1 \leq j \leq N} .
$$

Then the following items are equivalent.

(i). $E$ is an almost phaseless sampling sequence for $\left.V_{m}\right|_{\left[N_{1}, N_{2}\right]}$.

(ii). $\left\{D_{s} \Phi^{*} \mathbb{R}^{K}: s \in P_{N}\right\}$ consists of distinct $K$-dimensional subspaces.

(iii). $\left\{\mathcal{N}\left(\Phi D_{s}\right): s \in P_{N}\right\}$ consists of distinct $(N-K)$-dimensional subspaces.

Proof. The equivalence of (ii) and (iii) is obvious. We only need to show that (i) and (ii) are equivalent.

(i) $\Rightarrow$ (ii). Let $E$ be an almost phaseless sampling sequence. First, we show that $\operatorname{rank}(\Phi)=K$. Assume on the contrary that

$$
\operatorname{rank}(\Phi)<K
$$

Then we can find some $c \in R^{K} \backslash\{0\}$ such that $\Phi^{*} c=0$. Let

$$
f_{0}(x)=\sum_{n=N_{1}-m}^{N_{2}-1} c_{n} \varphi_{m}(x-n) .
$$

Then we have $f_{0} \neq 0$ and $f_{0}\left(x_{i}\right)=0$ for $1 \leq i \leq N$. Consequently, for $f \neq-(1 / 2) f_{0}$, we have $f \neq \pm\left(f+f_{0}\right)$ but $\left|f\left(x_{i}\right)\right|=\left|f\left(x_{i}\right)+f_{0}\left(x_{i}\right)\right|$. Hence we can not recover $f$ from intensity measurements, which contradicts with the assumption.

Set $F=\left(f\left(x_{1}\right), \ldots, f\left(x_{N}\right)\right)^{*}$ and $c=\left(c_{N_{1}-m}, \ldots, c_{N_{2}-1}\right)^{*}$. Then we have

$$
F=\Phi^{*} c .
$$

Since $E$ is an almost phaseless sampling sequence and $\left\{\left.\varphi_{m}(\cdot-n)\right|_{\left[N_{1}, N_{2}\right]}: N_{1}-m \leq n \leq\right.$ $\left.N_{2}-1\right\}$ is a basis for $\left.V_{m}\right|_{\left[N_{1}, N_{2}\right]}$, column vectors of $\Phi$ form an almost phase retrievable frame for $\mathbb{R}^{K}$. Now the conclusion follows from Theorem 2.3 ,

(ii) $\Rightarrow$ (i) can be proved similarly.

Lemma 2.8 An almost phaseless sampling sequence for $\left.V_{m}\right|_{\left[N_{1}, N_{2}\right]}$ is always a sampling sequence for $\left.V_{m}\right|_{\left[N_{1}, N_{2}\right]}$.

Proof. Let $E=\left\{x_{i}: 1 \leq i \leq N\right\}$ be an almost phaseless sampling sequence for $\left.V_{m}\right|_{\left[N_{1}, N_{2}\right]}$. Define $\Phi$ by (2.12). Set $F=\left(f\left(x_{1}\right), \ldots, f\left(x_{N}\right)\right)^{*}$ and $c=\left(c_{N_{1}-m}, \ldots, c_{N_{2}-1}\right)^{*}$. Then we have

$$
F=\Phi^{*} c
$$

We see from the proof of Lemma 2.7 that $\operatorname{rank}(\Phi)=N_{2}-N_{1}+m$. Hence $\Phi^{*} \Phi$ is invertible. Therefore,

$$
c=\left(\Phi \Phi^{*}\right)^{-1} \Phi F .
$$


It follows that

$$
f(x)=\sum_{n=N_{1}-m}^{N_{2}-1} c_{n} \varphi_{m}(x-n)=\sum_{k=i}^{N} f\left(x_{i}\right) S_{i}(x), \quad x \in\left[N_{1}, N_{2}\right],
$$

where $S_{i}$ is a linear combination of $\left\{\varphi_{m}(x-n): N_{1}-m \leq n \leq N_{2}-1\right\}$. In other words, $E$ is a local sampling sequence for $\left.V_{m}\right|_{\left[N_{1}, N_{2}\right]}$.

With the above lemma, we get the minimum cardinality of almost phaseless sampling sequences on $\left[N_{1}, N_{2}\right]$.

Corollary 2.9 Suppose that $E$ is an almost phaseless sampling sequence for $\left.V_{m}\right|_{\left[N_{1}, N_{2}\right]}$. Then we have $\# E \geq N_{2}-N_{1}+m+1$.

Proof. By Lemma 2.8, $E$ is a sampling sequence for $\left.V_{m}\right|_{\left[N_{1}, N_{2}\right]}$. Hence $\# E \geq N_{2}-$ $N_{1}+m$. If $\# E=N_{2}-N_{1}+m$, then the matrix $\Phi$ defined in (2.12) is an $\left(N_{2}-N_{1}+m\right) \times$ $\left(N_{2}-N_{1}+m\right)$ invertible matrix. By Lemma 2.7, $E$ is not a phaseless sampling sequence, which contradicts with the hypothesis. This completes the proof.

Next we show that every almost phaseless sampling sequence on $\left[N_{1}, N_{2}\right]$ contains a subsequence whose cardinality equals to the minimum $N_{2}-N_{1}+m+1$.

Lemma 2.10 Let $E \subset\left[N_{1}, N_{2}\right]$ be sequence of distinct numbers which meets (2.1)-2.4). Then there is a subsequence $E^{\prime} \subset E$ such that $\# E^{\prime}=N_{2}-N_{1}+m+1$ and $E^{\prime}$ meets (2.2) -2.4 .

Proof. First, we consider the case of $m=1$. We see from (2.2)-(2.4) that

$$
\begin{aligned}
& \#\left(E \cap\left[N_{1}, N_{1}+1\right)\right) \geq 2 \\
& \#\left(E \cap\left(N_{2}-1, N_{2}\right]\right) \geq 2, \\
& \#(E \cap(n, n+1)) \geq 1, \quad N_{1} \leq n \leq N_{2}-1 .
\end{aligned}
$$

Hence there is some $E^{\prime} \subset E$ such that

$$
\begin{aligned}
& \#\left(E^{\prime} \cap\left[N_{1}, N_{1}+1\right)\right)=2 \\
& \#\left(E^{\prime} \cap\left(N_{2}-1, N_{2}\right]\right)=2, \\
& \#\left(E^{\prime} \cap(n, n+1)\right)=1, \quad N_{1}<n<N_{2}-1 .
\end{aligned}
$$

Now we get a subsequence $E^{\prime}$ as desired.

Next we consider the case of $m \geq 2$. Set $K=\# E$. Assume that $K>N_{2}-N_{1}+m+1$. Let

$$
l_{k}=\#\left(E \cap\left[N_{1}, N_{1}+k\right)\right)-k-1, \quad 1 \leq k \leq N_{2}-N_{1} .
$$

Then we see from (2.2) that

$$
l_{k} \geq 0, \quad 1 \leq k \leq N_{2}-N_{1} .
$$


Set

$$
k_{0}=\min \left\{k \in\left[1, N_{2}-N_{1}\right]: l_{i} \geq 1, k \leq i \leq N_{2}-N_{1}\right\} .
$$

We conclude that

$$
\#\left(E \cap\left(N_{2}-k, N_{2}\right]\right) \geq k+2, \quad N_{2}-N_{1}-k_{0}+1 \leq k \leq N_{2}-N_{1} .
$$

Note that

$$
l_{N_{2}-N_{1}} \geq K-1-\left(N_{2}-N_{1}\right)-1 \geq m \geq 1 .
$$

We have $1 \leq k_{0} \leq N_{2}-N_{1}$. Since

$$
\#\left(E \cap\left(N_{1}, N_{2}\right]\right) \geq K-1 \geq N_{2}-N_{1}+m+1,
$$

(2.15) is true if $k_{0}=1$.

Next we suppose that $k_{0}>1$. We see from (2.14) that $l_{k_{0}-1}=0$. Hence

$$
\#\left(E \cap\left[N_{1}, N_{1}+k_{0}-1\right)\right)=k_{0} .
$$

Therefore,

$$
\#\left(E \cap\left[N_{1}+k_{0}-1, N_{2}\right]\right)=K-k_{0} .
$$

For $N_{2}-N_{1}-k_{0}+1<k \leq N_{2}-N_{1}$, we see from (2.4) and (2.16) that

$$
\begin{aligned}
& \#\left(E \cap\left(N_{2}-k, N_{2}\right]\right) \\
= & \#\left(E \cap\left(N_{2}-k, N_{1}+k_{0}-1\right)\right)+\#\left(E \cap\left[N_{1}+k_{0}-1, N_{2}\right]\right) \\
\geq & \left(N_{1}-N_{2}+k+k_{0}-m\right)+\left(K-k_{0}\right) \\
= & K-\left(N_{2}-N_{1}+m\right)+k \\
\geq & k+2 .
\end{aligned}
$$

And for $k=N_{2}-N_{1}-k_{0}+1$, we see from (2.16) that

$$
\begin{aligned}
\#\left(E \cap\left(N_{2}-k, N_{2}\right]\right) & \geq K-k_{0}-1=K-N_{2}+N_{1}-2+k \geq k+m \\
& \geq k+2 .
\end{aligned}
$$

Hence (2.15) is also true.

Next we show that

$$
\#\left(E \cap\left(N_{1}+k_{0}-1, N_{1}+k_{0}\right)\right) \geq 1 .
$$

If $k_{0}=1$, then (2.17) follows from (2.2). For the case of $k_{0}>1$, we see from (2.14) that $l_{k_{0}-1}=0$ and $l_{k} \geq 1$ for $k_{0} \leq k \leq N_{2}-N_{1}$. Hence

$$
\begin{aligned}
& \#\left(E \cap\left[N_{1}+k_{0}-1, N_{1}+k\right)\right) \\
= & \#\left(E \cap\left[N_{1}, N_{1}+k\right)\right)-\#\left(E \cap\left[N_{1}, N_{1}+k_{0}-1\right)\right) \\
\geq & k+2-k_{0}, \quad k_{0} \leq k \leq N_{2}-N_{1} .
\end{aligned}
$$


By setting $k=k_{0}$, we get

$$
\#\left(E \cap\left[N_{1}+k_{0}-1, N_{1}+k_{0}\right)\right) \geq 2 .
$$

Hence (2.17) is true.

Take some $y^{\prime} \in E \cap\left(N_{1}+k_{0}-1, N_{1}+k_{0}\right)$ and let $E^{\prime}=E \backslash\left\{y^{\prime}\right\}$. Then $E^{\prime}$ meets (2.1) $-(2.4)$.

Since $\# E^{\prime}=K-1 \geq N_{2}-N_{1}+m+1$, we need only to show that $E^{\prime}$ satisfies (2.2)-(2.4).

For $1 \leq k \leq k_{0}-1$, we have

$$
\#\left(E^{\prime} \cap\left[N_{1}, N_{1}+k\right)\right)=\#\left(E \cap\left[N_{1}, N_{1}+k\right)\right) \geq k+1 .
$$

And for $k_{0} \leq k \leq N_{2}-N_{1}$,

$$
\#\left(E^{\prime} \cap\left[N_{1}, N_{1}+k\right)\right)=\#\left(E \cap\left[N_{1}, N_{1}+k\right)\right)-1=l_{k}+k \geq k+1 .
$$

Hence (2.2) is true.

On the other hand, for $1 \leq k \leq N_{2}-N_{1}+k_{0}$, we have

$$
\begin{aligned}
\#\left(E^{\prime} \cap\left(N_{2}-k, N_{2}\right]\right) & =\#\left(E \cap\left(N_{2}-k, N_{2}\right]\right) \\
& \geq k+1 .
\end{aligned}
$$

And for $N_{2}-N_{1}+k_{0}+1 \leq k \leq N_{2}-N_{1}$, we see from (2.15) that

$$
\#\left(E^{\prime} \cap\left(N_{2}-k, N_{2}\right]\right)=\#\left(E \cap\left(N_{2}-k, N_{2}\right]\right)-1 \geq k+1 .
$$

Hence (2.3) is true.

Now it remains to prove that $E^{\prime}$ meets (2.4). There are three cases.

Case 1. $n_{1} \geq N_{1}+k_{0}$ or $n_{2} \leq N_{1}+k_{0}-1$. In this case, we have

$$
\#\left(E^{\prime} \cap\left(n_{1}, n_{2}\right)\right)=\#\left(E \cap\left(n_{1}, n_{2}\right)\right) \geq n_{2}-n_{1}-m+1 .
$$

Case 2. $n_{1}<N_{1}+k_{0}-1$ and $n_{2} \geq N_{1}+k_{0}$.

We see from (2.18) that for $k_{0} \leq k \leq N_{2}-N_{1}$,

$$
\#\left(E^{\prime} \cap\left[N_{1}+k_{0}-1, N_{1}+k\right)\right)=\#\left(E \cap\left[N_{1}+k_{0}-1, N_{1}+k\right)\right)-1 \geq k-k_{0}+1 .
$$

Hence, for $n_{1}<N_{1}+k_{0}-1$ and $n_{2} \geq N_{1}+k_{0}$,

$$
\begin{aligned}
\#\left(E^{\prime} \cap\left(n_{1}, n_{2}\right)\right) & =\#\left(E^{\prime} \cap\left(n_{1}, N_{1}+k_{0}-1\right)\right)+\#\left(E^{\prime} \cap\left[N_{1}+k_{0}-1, n_{2}\right)\right) \\
& \geq\left(N_{1}+k_{0}-1-n_{1}-m+1\right)+\left(n_{2}-N_{1}-k_{0}+1\right) \\
& =n_{2}-n_{1}-m+1 .
\end{aligned}
$$

Case 3. $n_{1}=N_{1}+k_{0}-1$ and $n_{2} \geq N_{1}+k_{0}$. By (2.18), we have

$$
\#\left(E^{\prime} \cap\left(n_{1}, n_{2}\right)\right)=\#\left(E \cap\left(n_{1}, n_{2}\right)\right)-1 \geq n_{2}-n_{1}-1 \geq n_{2}-n_{1}-m+1 .
$$

In all three cases, we show that $E^{\prime}$ meets (2.4). 
Repeating the previous arguments again and again, we get some $E^{\prime} \subset E$ such that $\# E^{\prime}=N_{2}-N_{1}+m+1$ and $E^{\prime}$ meets (2.2) - (2.4). This completes the proof.

We are now ready to give a proof for Theorem 2.2 .

Proof of Theorem 2.2. Denote $E=\left\{x_{i}: 1 \leq i \leq N\right\}$. Let $\Phi$ be defined by (2.12) and denote its column vectors by $\Phi_{1}, \ldots, \Phi_{N}$.

First, we prove the necessity. Assume that $E$ is an almost phaseless sampling sequence. By Corallory 2.9, $\# E \geq N_{2}-N_{1}+m+1$.

We see from Proposition 2.6 and Lemma 2.8 that (2.9)-(2.11) are true. If (2.2) or (2.3) is false, then we have

$$
\#\left(E \cap\left[N_{1}, N_{1}+k\right)\right)=k \quad \text { or } \quad \#\left(E \cap\left(N_{2}-k, N_{2}\right]\right)=k .
$$

If $\#\left(E \cap\left(n_{1}, n_{2}\right)\right) \leq n_{2}-n_{1}-m$ for some $n_{1}<n_{2}$, then $n_{2}-n_{1} \geq m$. We conclude that $E \cap\left(n_{1}, n_{2}\right) \neq \emptyset$. Otherwise, the matrix $\Phi^{*}$ has the following form,

$$
\Phi^{*}=\left(\begin{array}{cc}
A_{1} & 0 \\
0 & A_{2}
\end{array}\right)
$$

where $A_{1}$ is a $k_{1} \times\left(n_{1}-N_{1}+m\right)$ matrix, $A_{2}$ is a $k_{2} \times\left(N_{2}-n_{1}\right)$ matrix, and $k_{1}+k_{2}=$ $N$. Hence there exist some $s \neq s^{\prime}$ such that $D_{s} \Phi^{*} \mathbb{R}^{N_{2}-N_{1}+m}=D_{s^{\prime}} \Phi^{*} \mathbb{R}^{N_{2}-N_{1}+m}$. By Lemma 2.7, $E$ is not an almost phaseless sampling sequence, which contradicts with the assumption. Hence $\#\left(E \cap\left(n_{1}, n_{2}\right)\right) \geq 1$.

It follows from the above arguments that if $E$ does not meet one of (2.2)-(2.4), then there is some element in $E$, say $x_{N}$, such that $E \backslash\left\{x_{N}\right\}$ is not a sampling sequence.

Since $N-1 \geq N_{2}-N_{1}+m$, we see from the proof of Lemma 2.8 that $\operatorname{rank}\left(\Phi_{1}, \ldots, \Phi_{N-1}\right)$ $<N_{2}-N_{1}+m$. But $\operatorname{rank}(\Phi)=N_{2}-N_{1}+m$. Hence $\Phi_{N}$ is not a linear combination of $\Phi_{1}, \ldots, \Phi_{N-1}$. Therefore, if there exists some $\left(a_{1}, \ldots, a_{N}\right) \in \mathbb{R}^{N}$ such that

$$
\sum_{i=1}^{N} a_{i} \Phi_{i}=0,
$$

then we have $a_{N}=0$. It follows that for $s=(1, \ldots, 1,1)$ and $s^{\prime}=(1, \ldots, 1,-1), \Phi D_{s}$ and $\Phi D_{s^{\prime}}$ have the same null space. By Lemma 2.7, $E$ is not an almost phaseless sampling sequence, which contradicts with the assumption.

Next we prove the sufficiency. Suppose that $E$ meets (2.1)-(2.4). by Lemma2.10, there is a subsequence $E^{\prime} \subset E$ such that $K:=\# E^{\prime}=N_{2}-N_{1}+m+1$ and $E^{\prime}$ meets (2.2)-(2.4). We see from Proposition 2.6 that $E^{\prime}$ remains a local sampling sequence whenever any one of its elements is removed. Let $\Phi^{\prime}$ be defined similarly with (2.12). Then $\Phi^{\prime}$ is full spark. Hence there exists some $a \in \mathbb{R}^{K}$ with non-zero entries such that

$$
\Phi^{\prime} a=0 .
$$

It follows that for $s=\left(p_{1}, \ldots, p_{K}\right) \in P_{K}$, the null space of $\Phi^{\prime} D_{s}$ is $\left\{x D_{s} a: x \in \mathbb{R}\right\}$. Since entries of $a$ are non-zero, these null spaces are distinct. By Lemma 2.7, $E^{\prime}$ is an almost phaseless sampling sequence. Since $E^{\prime} \subset E$, we get the conclusion as desired. 


\section{Local Phaseless Sampling in Spline Spaces}

In this section, we give a proof of Theorem 1.2. We begin with a characterization of separable functions. The following result coincides with [13, Theorem II.6] and [14, Corollary 2.6], where the separability for functions in general shift invariant spaces was established.

Lemma 3.1 Let $N_{1}<N_{2}$ be integers. Then a function $\left.f \in V_{m}\right|_{\left[N_{1}, N_{2}\right]}$ is separable if and only if $N_{2}-N_{1} \geq 2$ and $f=\sum_{n=N_{1}-m}^{N_{2}-1} c_{n} \varphi_{m}(\cdot-n)$ for some $c_{N_{1}-m}, \ldots, c_{N_{2}-1} \in \mathbb{R}$ such that $c_{n_{1}} \neq 0, c_{n_{2}} \neq 0$ and $c_{n}=0, n_{1}<n<n_{2}$ for some $n_{1}, n_{2}$ with $n_{2}-n_{1} \geq m+1$.

Proof. Since $\left\{\left.\varphi_{m}(\cdot-n)\right|_{\left[N_{1}, N_{2}\right]}: N_{1}-m \leq n \leq N_{2}-1\right\}$ is a basis for $\left.V_{m}\right|_{\left[N_{1}, N_{2}\right]}$, for any $\left.f \in V_{m}\right|_{\left[N_{1}, N_{2}\right]}$, there exist some $c_{N_{1}-m}, \ldots, c_{N_{2}-1} \in \mathbb{R}$ such that $f=\sum_{n=N_{1}-m}^{N_{2}-1} c_{n} \varphi_{m}(\cdot-$ $n)$.

Necessity. Assume that $f$ is separable, that is, there exist $f_{1},\left.f_{2} \in V_{m}\right|_{\left[N_{1}, N_{2}\right]} \backslash\{0\}$ such that $f=f_{1}+f_{2}$ and $f_{1}(x) f_{2}(x)=0$ for all $x \in\left[N_{1}, N_{2}\right]$. For any $N_{1} \leq n<N_{2}$, since both $f_{1}$ and $f_{2}$ are polynomials on $[n, n+1]$, one of $f_{1}$ and $f_{2}$ must be identical to zero on $[n, n+1]$.

On the other hand, since $f_{1}, f_{2} \neq 0$, there exist integers $i_{1}$ and $i_{2}$ such that

$$
\left.f_{1}\right|_{\left[i_{2}, i_{2}+1\right]} \neq 0 \quad \text { and }\left.\quad f_{2}\right|_{\left[i_{1}, i_{1}+1\right]} \neq 0 .
$$

Consequently,

$$
\left.f_{1}\right|_{\left[i_{1}, i_{1}+1\right]}=0 \quad \text { and }\left.\quad f_{2}\right|_{\left[i_{2}, i_{2}+1\right]}=0 .
$$

Hence $N_{2}-N_{1} \geq 2$. Without loss of generality, assume that $i_{1}<i_{2}$.

Let

$$
i_{1}^{\prime}=\max \left\{i:\left.f_{1}\right|_{\left[i_{1}, i\right]}=0\right\} .
$$

Then we have $i_{1}<i_{1}^{\prime} \leq i_{2}$ and $\left.f_{1}\right|_{\left[i_{1}^{\prime}, i_{1}^{\prime}+1\right]} \neq 0$. Since $f_{1} f_{2}=0$, we have $\left.f_{2}\right|_{\left[i_{1}^{\prime}, i_{1}^{\prime}+1\right]}=0$.

Suppose that $f_{l}=\sum_{n=N_{1}-m}^{N_{2}-1} c_{n}^{(l)} \varphi_{m}(\cdot-n), l=1,2$. We see from (3.1) that

$$
c_{i_{1}-m}^{(1)}=\ldots=c_{i_{1}^{\prime}-1}^{(1)}=0 \quad \text { and } \quad c_{i_{1}^{\prime}}^{(1)} \neq 0 .
$$

On the other hand, we see from $\left.f_{2}\right|_{\left[i_{1}^{\prime}, i_{1}^{\prime}+1\right]}=0$ that

$$
c_{i_{1}^{\prime}-m}^{(2)}=\ldots=c_{i_{1}^{\prime}}^{(2)}=0 .
$$

Hence $c_{i_{1}^{\prime}} \neq 0$ and

$$
c_{i}=c_{i}^{(1)}+c_{i}^{(2)}=0, \quad i_{1}^{\prime}-m \leq i \leq i_{1}^{\prime}-1 .
$$

Since $\left.f_{2}\right|_{\left[i_{1}, i_{1}+1\right]} \neq 0$, there is some $i_{0}$ with $i_{1}-m \leq i_{0} \leq i_{1}<i_{1}^{\prime}$ such that

$$
c_{i_{0}}^{(2)} \neq 0
$$

It follows from (3.2) that $c_{i_{0}} \neq 0$. By (3.3),$i_{0}<i_{1}^{\prime}-m$. Set $n_{1}=\max \left\{n \in\left[N_{1}-m, i_{1}^{\prime}-\right.\right.$ $\left.m-1]: c_{n} \neq 0\right\}$ and $n_{2}=i_{1}^{\prime}$. Then we get the conclusion as desired. 
Sufficiency. Let $f$ meets the hypothesis. Set

$$
f_{1}=\sum_{n=N_{1}-m}^{n_{1}} c_{n} \varphi_{m}(\cdot-n) \text { and } f_{2}=\sum_{n=n_{2}}^{N_{2}-1} c_{n} \varphi_{m}(\cdot-n) .
$$

Then we have $f_{1}, f_{2} \neq 0$ and $f=f_{1}+f_{2}$. Since

$$
\operatorname{supp} f_{1} \subset\left[N_{1}, n_{1}+m+1\right], \quad \operatorname{supp} f_{2} \subset\left[n_{2}, N_{2}\right]
$$

and $n_{2}-n_{1} \geq m+1$, we have $f_{1} f_{2}=0$. This completes the proof.

We see from Proposition 2.6 that for a sequence $E \subset\left[N_{1}, N_{2}\right]$ to be a sampling sequence for $\left.V_{m}\right|_{\left[N_{1}, N_{2}\right]}$, it must satisfy four conditions (2.8)-(2.11). The following result shows that whenever $\# E \geq N_{2}-N_{1}+m$, it determines functions in $V_{m}$ locally.

Lemma 3.2 Suppose that $N_{1}<N_{2}$ are integers and $E \subset\left[N_{1}, N_{2}\right]$. If $\# E \geq N_{2}-N_{1}+m$, then there exist some integers $n_{1}, n_{2} \in\left[N_{1}, N_{2}\right]$ such that $n_{1}<n_{2}$ and $E \cap\left[n_{1}, n_{2}\right]$ is a sampling sequence for $\left.V_{m}\right|_{\left[n_{1}, n_{2}\right]}$.

Proof. We prove the conclusion with induction on $N_{2}-N_{1}$. We see from Proposition 2.6 that it is the case if $N_{2}-N_{1}=1$.

Now we assume that the conclusion is true whenever $1 \leq N_{2}-N_{1} \leq n$ for some $n \geq 1$. Let us consider the case of $N_{2}-N_{1}=n+1$. Assume that $E$ is not a sampling sequence for $\left.V_{m}\right|_{\left[N_{1}, N_{2}\right]}$. By Proposition 2.6, there are three cases.

(i). There is some $k \in\left[1, N_{2}-N_{1}\right]$ such that $\#\left(E \cap\left[N_{1}, N_{1}+k\right)\right)<k$.

In this case, we have $k<N_{2}-N_{1}$ and $\#\left(E \cap\left[N_{1}+k, N_{2}\right]\right) \geq N_{2}-N_{1}-k+m+1$. Now we see from the inductive assumption that there exist some integers $n_{1}, n_{2} \in\left[N_{1}+k, N_{2}\right]$ such that $n_{1}<n_{2}$ and $E \cap\left[n_{1}, n_{2}\right]$ is a sampling sequence for $\left.V_{m}\right|_{\left[n_{1}, n_{2}\right]}$.

(ii). There is some $k \in\left[1, N_{2}-N_{1}\right]$ such that $\#\left(E \cap\left(N_{2}-k, N_{2}\right]\right)<k$.

Similarly to the previous case we can prove the conclusion.

(iii). $\#\left(E \cap\left[N_{1}, N_{1}+k\right)\right) \geq k$ and $\#\left(E \cap\left(N_{2}-k, N_{2}\right]\right) \geq k$ for any $1 \leq k \leq N_{2}-N_{1}$.

If for any integers $n_{1}<n_{2}$ with $\left[n_{1}, n_{2}\right] \subset\left[N_{1}, N_{2}\right]$, we have $\#\left(E \cap\left(n_{1}, n_{2}\right)\right) \geq$ $n_{2}-n_{1}-m$, then we see from Proposition 2.6 that $E$ is a sampling sequence for $\left.V_{m}\right|_{\left[N_{1}, N_{2}\right]}$. If $\#\left(E \cap\left(n_{1}, n_{2}\right)\right) \leq n_{2}-n_{1}-m-1$ for some $n_{1}<n_{2}$, then we have $n_{2}-n_{1} \geq m+1$ and $\left(n_{1}, n_{2}\right) \neq\left(N_{1}, N_{2}\right)$. Without loss of generality, we assume that $N_{1}<n_{1}$. Then

$$
\begin{aligned}
\#\left(E \cap\left[N_{1}, n_{1}\right]\right) & =\#\left(E \cap\left[N_{1}, n_{2}\right)\right)-\#\left(E \cap\left(n_{1}, n_{2}\right)\right) \\
& \geq\left(n_{2}-N_{1}\right)-\left(n_{2}-n_{1}-m-1\right) \geq n_{1}-N_{1}+m+1 .
\end{aligned}
$$

By the inductive assumption, there exist some integers $i_{1}<i_{2}$ in $\left[N_{1}, n_{1}\right]$ such that $E \cap\left[i_{1}, i_{2}\right]$ is a sampling sequence for $\left.V_{m}\right|_{\left[i_{1}, i_{2}\right]}$.

By induction, the conclusion is true for any $N_{1}<N_{2}$.

To prove Theorem 1.2, we need the following result on the invertibility of submatrix of (2.12).

Proposition 3.3 ( [26, Theorem 4.65]) Let $n_{1}<\ldots<n_{r}$ be $r$ integers and $t_{1}<$ $\ldots<t_{r}$ be $r$ real numbers. Then the matrix $\left[\varphi_{m}\left(t_{i}-n_{j}\right)\right]_{1 \leq i, j \leq r}$ is invertible if and only if $\varphi_{m}\left(t_{i}-n_{i}\right) \neq 0$ for $1 \leq i \leq r$. 
Proof of the necessity of Theorem 1.2. Denote $E=\left\{x_{i}: 1 \leq i \leq N\right\}$ and

$$
J_{k}=\#\left(E \cap\left[N_{1}, N_{1}+k\right)\right), \quad 1 \leq k \leq N_{2}-N_{1} .
$$

First, we prove that

$$
\begin{aligned}
& \#\left(E \cap\left[N_{1}, N_{1}+1\right)\right) \geq m+1, \\
& \#\left(E \cap\left(N_{2}-1, N_{2}\right]\right) \geq m+1 .
\end{aligned}
$$

Assume on the contrary that $J_{1} \leq m$. Define

$$
\begin{aligned}
& A_{1}=\left[\varphi_{m}\left(x_{i}-n\right)\right]_{1 \leq i \leq J_{1}, N_{1}-m \leq n \leq N_{2}-1}, \\
& A_{2}=\left[\varphi_{m}\left(x_{i}-n\right)\right]_{J_{1}+1 \leq i \leq N, N_{1}-m \leq n \leq N_{2}-1} .
\end{aligned}
$$

Note that $\operatorname{rank}\left(A_{1}\right) \leq J_{1} \leq m$ and the last $N_{2}-N_{1}-1$ columns of $A_{1}$ are zeros. There is a solution $c=\left(c_{N_{1}-m}, \ldots, c_{N_{2}-1}\right)^{*} \in \mathbb{R}^{N_{2}-N_{1}+m}$ of the equation

$$
A_{1} c=0
$$

such that $c_{N_{1}+1}=\ldots=c_{N_{2}-1}=1$ and not all of $c_{N_{1}-m}, \ldots, c_{N_{1}}$ are zeros. Since the first column of $A_{1}$ is not zero, not all of $c_{N_{1}-m+1}, \ldots, c_{N_{1}}$ are zeros.

Let $c^{\prime}=\left(1+\left|c_{N_{1}-m}\right|, 0, \ldots, 0\right)^{*}$. Then we have

$$
A_{2} c^{\prime}=0 .
$$

Let

$$
\left\{\begin{array}{l}
f_{1}=\sum_{n=N_{1}-m}^{N_{2}-1} \frac{1}{2}\left(c_{n}+c_{n}^{\prime}\right) \varphi_{m}(\cdot-n), \\
f_{2}=\sum_{n=N_{1}-m}^{N_{2}-1} \frac{1}{2}\left(c_{n}-c_{n}^{\prime}\right) \varphi_{m}(\cdot-n) .
\end{array}\right.
$$

Since there are at most $m-1$ entries of $c \pm c^{\prime}$ are zeros, by Lemma 3.1, both $f_{1}$ and $f_{2}$ are nonseparable. Moreover, $f_{1} \pm f_{2} \neq 0$ and

$$
\begin{aligned}
& f_{1}\left(x_{i}\right)=-f_{2}\left(x_{i}\right), \quad 1 \leq i \leq J_{1}, \\
& f_{1}\left(x_{i}\right)=f_{2}\left(x_{i}\right), \quad J_{1}+1 \leq i \leq N .
\end{aligned}
$$

Hence we can not recover $f$ from unsigned samples, which contradicts with the hypothesis. This proves (3.4). Similarly we can prove (3.5).

Next we prove (1.4). We begin with the simple case $n_{2}-n_{1}=1$. We have to show that

$$
\#(E \cap(n, n+1)) \geq 1, \quad N_{1} \leq n \leq N_{2}-1 .
$$

We see from (3.4) and (3.5) that (3.9) is true for $n=N_{1}$ or $N_{2}-1$. Now assume that $\#(E \cap(n, n+1))=0$ for some $n \in\left[N_{1}+1, N_{2}-2\right]$. Define

$$
A_{1}=\left[\varphi_{m}\left(x_{i}-j\right)\right]_{x_{i} \leq n, N_{1}-m \leq j \leq N_{2}-1},
$$




$$
A_{2}=\left[\varphi_{m}\left(x_{i}-j\right)\right]_{x_{i} \geq n+1, N_{1}-m \leq j \leq N_{2}-1} .
$$

Let $c=(0, \ldots, 0,1, \ldots, 1)^{*}$ and $c^{\prime}=(1, \ldots, 1,0, \ldots, 0)^{*}$, where the first $n-N_{1}+m$ entries of $c$ and the last $N_{2}-n+m-1$ entries of $c^{\prime}$ are zeros and the rest are 1 . Then we have

$$
A_{1} c=A_{2} c^{\prime}=0 .
$$

Let $f_{1}$ and $f_{2}$ be defined by (3.8). Since there are only $m-1$ entries of $c \pm c^{\prime}$ are zeros, with similar arguments we get a contradiction. Hence (3.9) is true.

Now we assume that (1.4) is false. Then there are integers $i_{1}<i_{2}$ with $i_{2}-i_{1} \geq 2$ such that $\#\left(E \cap\left(i_{1}, i_{2}\right)\right) \leq 2\left(i_{2}-i_{1}\right)-2$. Let

$$
\begin{array}{r}
n_{2}=\min \left\{n \in\left[N_{1}, N_{2}\right]: \text { there exists some } n_{1}<n\right. \text { such that } \\
\left.\#\left(E \cap\left(n_{1}, n\right)\right) \leq 2\left(n-n_{1}\right)-2\right\}, \\
n_{1}=\max \left\{n \in\left[N_{1}, n_{2}\right): \#\left(E \cap\left(n, n_{2}\right)\right) \leq 2\left(n_{2}-n\right)-2\right\} .
\end{array}
$$

Then we have

$$
\begin{aligned}
& n_{1} \leq n_{2}-2, \\
& \#\left(E \cap\left(n_{1}, n_{1}+1\right)\right)=\#\left(E \cap\left(n_{1}, n_{1}+1\right]\right)=1, \\
& \#\left(E \cap\left(n_{2}-1, n_{2}\right)\right)=\#\left(E \cap\left[n_{2}-1, n_{2}\right)\right)=1, \\
& \#(E \cap[n, n+1))=\#(E \cap(n, n+1])=2, n_{1}+1 \leq n \leq n_{2}-2, \\
& E \cap\left(n_{1}, n_{2}\right) \cap \mathbb{Z}=\emptyset .
\end{aligned}
$$

Moreover, we see from the definitions of $n_{1}, n_{2}$ and (3.13) that

$$
\begin{aligned}
\#(E \cap(n, n+2)) & \geq 3, & & N_{1} \leq n \leq n_{1}-1, \\
\#\left(E \cap\left(n, n_{1}\right]\right) & \geq 2\left(n_{1}-n\right), & & N_{1} \leq n \leq n_{1}-1 .
\end{aligned}
$$

It follows that if $n_{1}>N_{1}+1$, then

$$
\begin{aligned}
\#\left(E \cap\left[N_{1}, n_{2}\right)\right)= & \#\left(E \cap\left[N_{1}, N_{1}+1\right]\right)+\#\left(E \cap\left(N_{1}+1, n_{1}\right]\right) \\
& \quad+\#\left(E \cap\left(n_{1}, n_{2}\right)\right) \\
\geq & 2\left(n_{2}-N_{1}\right)+m-3 .
\end{aligned}
$$

And the above inequality is also true if $n_{1} \leq N_{1}+1$.

By (3.9), there is some $E_{2} \subset E$ such that

$$
\begin{aligned}
& E \cap\left[n_{2}, N_{2}\right] \subset E_{2}, \\
& \#\left(E_{2} \cap\left[N_{1}, n_{2}\right)\right)=n_{2}-N_{1}-1, \\
& \#\left(E_{2} \cap(n, n+1)\right)=1, \quad N_{1} \leq n \leq n_{2}-2 .
\end{aligned}
$$

Let $E_{1}=E \backslash E_{2}$. Define

$$
A_{1}=\left[\varphi_{m}\left(x_{i}-l\right)\right]_{x_{i} \in E_{1}, N_{1}-m \leq l \leq N_{2}-1},
$$




$$
A_{2}=\left[\varphi_{m}\left(x_{i}-l\right)\right]_{x_{i} \in E_{2}, N_{1}-m \leq l \leq N_{2}-1}
$$

Observe that $E_{2} \cap\left[n_{2}-1, n_{2}\right)=\emptyset$. The first $n_{2}-N_{1}$ columns of $A_{2}$ has the form $\left(\begin{array}{c}A_{21} \\ 0\end{array}\right)$, where

$$
A_{21}=\left(\begin{array}{ccccccc}
* & * & ? & \ldots & & & \\
0 & * & * & \ldots & & & \\
0 & 0 & * & \ldots & & & \\
& \ldots & & & \ldots & & \\
0 & 0 & 0 & \ldots & * & * & ? \\
0 & 0 & 0 & \ldots & 0 & * & *
\end{array}\right)
$$

is an $\left(n_{2}-N_{1}-1\right) \times\left(n_{2}-N_{1}\right)$ matrix. Here ' $*$ ' stands for a nonzero entry and '?' means uncertainty (it is 0 if $m=1$ ). By Proposition 3.3, every $\left(n_{2}-N_{1}-1\right) \times\left(n_{2}-N_{1}-1\right)$ submatrix of $A_{21}$ is invertible. Hence there exists some $c \in \mathbb{R}^{n_{2}-N_{1}}$ such that none of its entries is zero and

$$
A_{21} c=0 .
$$

By appending $N_{2}-n_{2}+m$ zeros to $c$ we get a $c^{\prime} \in \mathbb{R}^{N_{2}-N_{1}+m}$, none of whose first $n_{2}-N_{1}$ entries is zero, such that

$$
A_{2} c^{\prime}=0 .
$$

On the other hand, since $E_{1} \subset\left[N_{1}, n_{2}\right)$, we have $A_{1}=\left(A_{11}, 0\right)$, where $A_{11}$ has $n_{2}-N_{1}+m$ columns and at least $n_{2}-N_{1}+m-2 \geq n_{2}-n_{1}+m-2$ rows. Note that

$$
\#\left(E_{1} \cap\left(n_{1}, n_{1}+1\right]\right)=0 \text { and } \#\left(E_{1} \cap(n, n+1]\right)=1 \text { for } n_{1}+1 \leq n \leq n_{2}-1 \text {. }
$$

$A_{11}$ has the following form,

$$
A_{11}=\left(\begin{array}{cccccccc}
\ldots & & & & \ldots & & & \\
\ldots & 0 & 0 & 0 & \ldots & & & \\
\ldots & * & * & 0 & \ldots & & & \\
\ldots & & \ldots & & \ldots & & & \\
\ldots & & & & \ldots & * & * & 0 \\
\ldots & & & & \ldots & ? & * & *
\end{array}\right)
$$

where the submatrix consisting of the last $n_{2}-n_{1}$ columns of $A_{11}$ has only $n_{2}-n_{1}-1$ non-zero rows. Using Proposition 3.3 again, we get some $\tilde{c} \in \mathbb{R}^{n_{2}-N_{1}+m}$ such that the first $n_{1}-N_{1}+m$ entries of $\tilde{c}$ are zeros, none of the last $n_{2}-n_{1}$ entries is zero, and $A_{11} \tilde{c}=0$. Consequently, there is some $c=\left(c_{N_{1}-m}, \ldots, c_{N_{2}-1}\right)^{*} \in \mathbb{R}^{N_{2}-N_{1}+m}$ such that

$$
c_{i}=0, i \leq n_{1}-1 \text { or } i \geq n_{2}, \quad c_{i} \neq 0, n_{1} \leq i \leq n_{2}-1,
$$

and

$$
A_{1} c=0 .
$$

By multiplying a factor we can suppose that

$$
\min \left\{\left|c_{i}^{\prime}\right|:\left|c_{i}^{\prime}\right|>0\right\}>\max \left\{\left|c_{i}\right|:\left|c_{i}\right|>0\right\} .
$$


Then we have

$$
\begin{aligned}
c_{i} \pm c_{i}^{\prime} & \neq 0, \quad i \leq n_{2}-m-1 \\
c_{n_{2}-1} \pm c_{n_{2}-1}^{\prime} & \neq 0, \\
c_{i} \pm c_{i}^{\prime} & =0, \quad i \geq n_{2} .
\end{aligned}
$$

Let $f_{1}$ and $f_{2}$ be defined by (3.8). With similar arguments we get a contradiction. Hence (1.4) is true.

Next we prove (1.2). Assume on the contrary that (1.2) is false. Since $J_{1} \geq m+1$, there is some $k_{0} \geq 2$ such that $J_{k_{0}} \leq 2 k_{0}+m-2$ and $J_{i} \geq 2 i+m-1$ for $1 \leq i \leq k_{0}-1$.

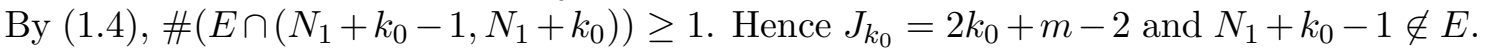

As in the above arguments, we can split $E$ into two subsequences $E_{1}$ and $E_{2}$ which satisfy (3.19) - (3.21) with $n_{2}$ being replaced by $N_{1}+k_{0}$. Define $A_{1}$ and $A_{2}$ by (3.22) and (3.23), respectively. Then there exists some $c^{\prime} \in \mathbb{R}^{N_{2}-N_{1}+m}$, for which the first $k_{0}$ entries are nonzero and the last $N_{2}-N_{1}-k_{0}+m$ entries are zeros, such that

$$
A_{2} c^{\prime}=0
$$

On the other hand, since $\# E_{1}=k_{0}+m-1$, by Proposition 2.6, $E_{1}$ is not a sampling sequence for $\left.V_{m}\right|_{\left[N_{1}, N_{1}+k_{0}\right]}$. Hence there is some $c \in \mathbb{R}^{N_{2}-N_{1}+m} \backslash\{0\}$, whose last $N_{2}-N_{1}-k_{0}$ entries are zeros, such that

$$
A_{1} c=0 .
$$

Again, we assume that (3.24) holds. With similar arguments we get a contradiction. Hence (1.2) is true. Similarly we can prove (1.3).

Finally, we prove (1.1). Assume on the contrary that \#E $\leq 2\left(N_{2}-N_{1}+m\right)-2$. By Lemma 2.8, $\# E \geq N_{2}-N_{1}+m$. Take some $E_{1} \subset E$ such that $\# E_{1}=N_{2}-N_{1}+m-1$ and

$$
\#\left(E_{1} \cap(n, n+1)\right) \geq 1, \quad N_{1} \leq n \leq N_{2}-1 .
$$

We see from (1.4) that such $E_{1}$ exists. Let $E_{2}=E \backslash E_{1}$. Define $A_{1}$ and $A_{2}$ by (3.22) and (3.23), respectively. Since $\operatorname{rank}\left(A_{1}\right) \leq N_{2}-N_{1}+m-1$, there is some $c \in \mathbb{R}^{N_{2}-N_{1}+m} \backslash\{0\}$ such that

$$
A_{1} c=0 .
$$

We conclude that there is not an integer $i$ such that $c_{i}=\ldots=c_{i+m-1}=0$. Otherwise, we see from (3.25) that $c_{l}=0$ for all $N_{1}-m \leq l \leq N_{2}-1$, which contradicts with $c \neq 0$.

On the other hand, since $\#\left(E \backslash E_{1}\right) \leq N_{2}-N_{1}+m-1$, the equation

$$
A_{2} c^{\prime}=0
$$

has a non-zero solution. Again, we assume that (3.24) holds. With similar arguments we get a contradiction. This completes the proof of the necessity.

To prove the sufficiency of Theorem 1.2, we first present some preliminary results. 
Lemma 3.4 Let $n_{1}<n_{1}^{\prime} \leq n_{2}<n_{2}^{\prime}$ be integers and $E \subset\left[n_{1}, n_{2}^{\prime}\right]$ be a sequence consisting of distinct points such that $\#(E \cap(i, j)) \geq 2(j-i)-1$ whenever $n_{1} \leq i<j \leq n_{2}^{\prime}$. Suppose that $E_{1} \cup E_{2}=E, E_{1} \cap E_{2}=\emptyset$ and that $E_{l} \cap\left[n_{l}, n_{l}^{\prime}\right] \geq n_{l}^{\prime}-n_{l}+m, l=1,2$. Then there exist integers $i_{1}<i_{1}^{\prime}=i_{2}<i_{2}^{\prime}$ in $\left[n_{1}, n_{2}^{\prime}\right]$ such that $E_{l} \cap\left[i_{l}, i_{l}^{\prime}\right]$ is a sampling sequence for $\left.V_{m}\right|_{\left[i_{l}, i_{l}^{\prime}\right]}, l=1,2$.

Proof. By Lemma 3.2, we may assume that $E_{l} \cap\left[n_{l}, n_{l}^{\prime}\right]$ is a sampling sequence for $\left.V_{m}\right|_{\left[n_{l}, n_{l}^{\prime}\right]}, l=1,2$. Note that $n_{1}^{\prime} \leq n_{2}$. Let $i_{1}^{\prime}$ be the maximum of all integers $i \in\left(n_{1}, n_{2}\right]$ such that for some $i_{1} \in\left[n_{1}, i\right), E_{1} \cap\left[i_{1}, i_{1}^{\prime}\right]$ is a sampling sequence for $\left.V_{m}\right|_{\left[i_{1}, i_{1}^{\prime}\right]}$. And let $i_{2}$ be the minimum of all integers $i \in\left[i_{1}^{\prime}, n_{2}^{\prime}\right)$ such that for some $i_{2}^{\prime} \in\left(i_{2}, n_{2}^{\prime}\right], E_{2} \cap\left[i_{2}, i_{2}^{\prime}\right]$ is a sampling sequence for $\left.V_{m}\right|_{\left[i_{2}, i_{2}^{\prime}\right]}$. We conclude that $i_{1}^{\prime}=i_{2}$.

Assume on the contrary that $i_{1}^{\prime}<i_{2}$. Since $\#\left(E \cap\left(i_{1}^{\prime}, i_{2}\right)\right) \geq 2\left(i_{2}-i_{1}^{\prime}\right)-1$, one of $\#\left(E_{1} \cap\left(i_{1}^{\prime}, i_{2}\right)\right)$ and $\#\left(E_{2} \cap\left(i_{1}^{\prime}, i_{2}\right)\right)$ must be no less than $i_{2}-i_{1}^{\prime}$. Without loss of generality, we assume that $\#\left(E_{2} \cap\left(i_{1}^{\prime}, i_{2}\right)\right) \geq i_{2}-i_{1}^{\prime}$. Let

$$
j_{2}^{\prime}=\max \left\{j<i_{2}: \#\left(E_{2} \cap\left(j, i_{2}\right)\right) \geq i_{2}-j\right\} .
$$

Then we have $i_{1}^{\prime} \leq j_{2}^{\prime}<i_{2}$. For $j_{2}^{\prime}<j<i_{2}$, since

$$
\#\left(E_{2} \cap\left(j, i_{2}\right)\right) \leq i_{2}-j-1,
$$

we have

$$
\#\left(E_{2} \cap\left(j_{2}^{\prime}, j\right]\right) \geq j-j_{2}^{\prime}+1 .
$$

Hence

$$
\#\left(E_{2} \cap\left(j_{2}^{\prime}, j\right)\right) \geq j-j_{2}^{\prime}, \quad j_{2}^{\prime}<j \leq i_{2} .
$$

On the other hand, since $E_{2} \cap\left[i_{2}, i_{2}^{\prime}\right]$ is a sampling sequence for $\left.V_{m}\right|_{\left[i_{2}, i_{2}^{\prime}\right]}$, we have

$$
\#\left(E_{2} \cap\left(j_{2}^{\prime}, j\right)\right) \geq j-j_{2}^{\prime}, \quad j_{2}^{\prime}<j \leq i_{2}^{\prime} .
$$

By the choice of $i_{2}, E_{2} \cap\left[j_{2}^{\prime}, i_{2}^{\prime}\right]$ is not a sampling sequence for $\left.V_{m}\right|_{\left[j_{2}^{\prime}, i_{2}^{\prime}\right]}$. Since

$$
\begin{aligned}
\#\left(E_{2} \cap\left(j_{2}^{\prime}, i_{2}^{\prime}\right]\right) & =\#\left(E_{2} \cap\left(j_{2}^{\prime}, i_{2}\right)\right)+\#\left(E_{2} \cap\left[i_{2}, i_{2}^{\prime}\right]\right) \\
& \geq i_{2}^{\prime}-j_{2}^{\prime}+m,
\end{aligned}
$$

by Proposition 2.6, there are two cases.

(a) There exist integers $l, l^{\prime} \in\left[j_{2}^{\prime}, i_{2}^{\prime}\right]$ such that $l<l^{\prime}$ and $\#\left(E_{2} \cap\left(l, l^{\prime}\right)\right) \leq l^{\prime}-l-m-1$.

Recall that $E_{2} \cap\left[i_{2}, i_{2}^{\prime}\right]$ is a sampling sequence for $\left.V_{m}\right|_{\left[i_{2}, i_{2}^{\prime}\right]}$. We have $l<i_{2}$. First, we assume that $l^{\prime}>i_{2}$. Since $\#\left(E_{2} \cap\left[i_{2}, l^{\prime}\right)\right) \geq l^{\prime}-i_{2}$, we have

$$
\#\left(E_{2} \cap\left(l, i_{2}\right)\right) \leq i_{2}-l-m-1 .
$$

It follows from (3.27) that

$$
\#\left(E_{2} \cap\left(j_{2}^{\prime}, l\right)\right) \geq l-j_{2}^{\prime}+m .
$$


By Lemma 3.2, there exist integers $l_{2}, l_{2}^{\prime} \in\left[j_{2}^{\prime}, l\right]$ such that $l_{2}<l_{2}^{\prime}$ and $E_{2} \cap\left[l_{2}, l_{2}^{\prime}\right]$ is sampling sequence for $\left.V_{m}\right|_{\left[l_{2}, l_{2}^{\prime}\right]}$, which contradicts with the choice of $i_{2}$.

Next we consider the case of $l^{\prime} \leq i_{2}$. We see from (3.26) that

$$
\begin{aligned}
\#\left(E_{2} \cap\left(l, i_{2}\right)\right) & =\#\left(E_{2} \cap\left(l, l^{\prime}\right)\right)+\#\left(E_{2} \cap\left[l^{\prime}, i_{2}\right)\right) \\
& \leq l^{\prime}-l-m-1+i_{2}-l^{\prime}=i_{2}-l-m-1 .
\end{aligned}
$$

With similar arguments we get a contradiction.

(b). There exists some inter $l \in\left[j_{2}^{\prime}, i_{2}^{\prime}\right)$ such that $\#\left(E_{2} \cap\left(l, i_{2}^{\prime}\right]\right) \leq i_{2}^{\prime}-l-1$.

In this case, we have $l<i_{2}$ and

$$
\begin{aligned}
\#\left(E \cap\left(l, i_{2}\right)\right) & =\#\left(E \cap\left(l, i_{2}^{\prime}\right]\right)-\#\left(E \cap\left[i_{2}, i_{2}^{\prime}\right]\right) \\
& \leq i_{2}-l-m-1 .
\end{aligned}
$$

Again, with similar arguments we get a contradiction. This completes the proof.

The following is a simple application of Lemma 3.4 .

Lemma 3.5 Let $E \subset\left[N_{1}, N_{2}\right]$ be a sequence consisting of distinct points which satisfies (1.1) - (1.4). Suppose that $E_{1} \cup E_{2}=E, E_{1} \cap E_{2}=\emptyset$, \# $E_{1} \leq \# E_{2}$, and that $E_{2}$ is not a sampling sequence for $\left.V_{m}\right|_{\left[N_{1}, N_{2}\right]}$. Then there exist integers $i_{1}, i_{1}^{\prime}, i_{2}, i_{2}^{\prime} \in\left[N_{1}, N_{2}\right]$ such that $i_{1}<i_{1}^{\prime}, i_{2}<i_{2}^{\prime},\left[i_{1}, i_{1}^{\prime}\right]$ and $\left[i_{2}, i_{2}^{\prime}\right]$ have and only have one common point, and $E_{l} \cap\left[i_{l}, i_{l}^{\prime}\right]$ is a sampling sequence for $\left.V_{m}\right|_{\left[i_{l}, i_{l}^{\prime}\right]}, l=1,2$.

Proof. Since $\# E_{2} \geq \# E_{1}$, we see from (1.1) that

$$
\# E_{2} \geq N_{2}-N_{1}+m \text {. }
$$

Since $E_{2}$ is not a sampling sequence for $\left.V_{m}\right|_{\left[N_{1}, N_{2}\right]}$, by Proposition 2.6, there are three cases.

(i) There is some integer $k \in\left[1, N_{2}-N_{1}\right]$ such that $\#\left(E_{2} \cap\left[N_{1}, N_{1}+k\right)\right) \leq k-1$.

In this case, we see from (1.2) that

$$
\begin{aligned}
\#\left(E_{1} \cap\left[N_{1}, N_{1}+k\right)\right) & =\#\left(E \cap\left[N_{1}, N_{1}+k\right)\right)-\#\left(E_{2} \cap\left[N_{1}, N_{1}+k\right)\right) \\
& \geq k+m .
\end{aligned}
$$

By Lemma 3.2, there exist integers $n_{1}, n_{1}^{\prime}$ such that $N_{1} \leq n_{1}<n_{1}^{\prime} \leq N_{1}+k$ and $E_{1} \cap\left[n_{1}, n_{1}^{\prime}\right]$ is a sampling sequence for $\left.V_{m}\right|_{\left[n_{1}, n_{1}^{\prime}\right]}$.

On the other hand, since

$$
\begin{aligned}
\#\left(E_{2} \cap\left[N_{1}+k, N_{2}\right]\right) & =\#\left(E_{2} \cap\left[N_{1}, N_{2}\right]\right)-\#\left(E_{2} \cap\left[N_{1}, N_{1}+k\right)\right) \\
& \geq N_{2}-N_{1}-k+m+1,
\end{aligned}
$$

Using Lemma 3.2 again, we get some integers $n_{2}, n_{2}^{\prime}$ with $N_{1}+k \leq n_{2}<n_{2}^{\prime} \leq N_{2}$ such that $E_{2} \cap\left[n_{2}, n_{2}^{\prime}\right]$ is a sampling sequence for $\left.V_{m}\right|_{\left[n_{2}, n_{2}^{\prime}\right]}$. By Lemma 3.4, we get the conclusion as desired.

(ii) There is some integer $k \in\left[1, N_{2}-N_{1}\right]$ such that $\#\left(E_{2} \cap\left(N_{2}-k, N_{2}\right]\right) \leq k-1$. 
Similarly to the first case we can prove the conclusion.

(iii) There exist integers $k_{1}, k_{2} \in\left[N_{1}, N_{2}\right]$ such that $k_{1}<k_{2}$ and $\#\left(E_{2} \cap\left(k_{1}, k_{2}\right)\right) \leq$ $k_{2}-k_{1}-m-1$.

In this case, we have

$$
\#\left(E_{1} \cap\left(k_{1}, k_{2}\right)\right) \geq k_{2}-k_{1}+m .
$$

Hence there exist integers $n_{1}, n_{1}^{\prime} \in\left[k_{1}, k_{2}\right]$ such that $n_{1}<n_{1}^{\prime}$ and $E_{1} \cap\left[n_{1}, n_{1}^{\prime}\right]$ is a sampling sequence for $\left.V_{m}\right|_{\left[n_{1}, n_{1}^{\prime}\right]}$.

On the other hand, If $\#\left(E_{2} \cap\left[N_{1}, k_{1}\right]\right) \leq k_{1}-N_{1}+m-1$ and $\#\left(E_{2} \cap\left[k_{2}, N_{2}\right]\right) \leq$ $N_{2}-k_{2}+m-1$, then

$$
\#\left(E_{2} \cap\left[N_{1}, N_{2}\right]\right) \leq N_{2}-N_{1}+m-3,
$$

which contradicts with (3.29). Hence either \#( $\left.E_{2} \cap\left[N_{1}, k_{1}\right]\right) \geq k_{1}-N_{1}+m$ or \# $\left(E_{2} \cap\right.$ $\left.\left[k_{2}, N_{2}\right]\right) \geq N_{2}-k_{2}+m$. Consequently, we can find some integers $n_{2}<n_{2}^{\prime}$ such that $\left[n_{2}, n_{2}^{\prime}\right] \subset\left[N_{1}, k_{1}\right]$ or $\left[n_{2}, n_{2}^{\prime}\right] \subset\left[k_{2}, N_{2}\right]$ and $E_{2} \cap\left[n_{2}, n_{2}^{\prime}\right]$ is a sampling sequence for $\left.V_{m}\right|_{\left[n_{2}, n_{2}^{\prime}\right]}$. Again, the conclusion follows from Lemma 3.4.

We finish the proof of Theorem 1.2 by presenting a slightly stronger result, of which the sufficiency of Theorem 1.2 is a consequence.

Lemma 3.6 Suppose that $E=\left\{x_{i}: 1 \leq i \leq N\right\}$ satisfies (1.1) - (1.4). Let $f_{i}=$ $\sum_{n=N_{1}-m}^{N_{2}-1} c_{n}^{(i)} \varphi_{m}(\cdot-n) \in V_{m}(i=1,2)$ be such that

$$
\left|f_{1}\left(x_{k}\right)\right|=\left|f_{2}\left(x_{k}\right)\right|, \quad 1 \leq k \leq N .
$$

Then $\left|f_{1}(x)\right|=\left|f_{2}(x)\right|$ on $\left[N_{1}, N_{2}\right]$. Moreover, if $f_{1} \neq \pm f_{2}$, then there exist integers $n_{1}, n_{2} \in\left[N_{1}, N_{2}\right]$ such that $n_{2}-n_{1} \geq m+1$,

$$
c_{n}^{(i)}=0, \quad n_{1}<n<n_{2}, i=1,2,
$$

and there exist $j_{1}^{(1)}, j_{1}^{(2)} \leq n_{1}$ and $j_{2}^{(1)}, j_{2}^{(2)} \geq n_{2}$ satisfying that none of $c_{j_{1}}^{(1)}, c_{j_{2}}^{(1)}, c_{j_{1}}^{(2)}$ and $c_{j_{2}}^{(2)}$ is zero.

Proof. Suppose that $f_{1} \pm f_{2} \neq 0$. Split $E$ into two subsequences $E_{1}$ and $E_{2}$ such that

$$
E_{1}=\left\{x_{i}: f_{1}\left(x_{i}\right)=-f_{2}\left(x_{i}\right)\right\} \quad \text { and } \quad E_{2}=\left\{x_{i}: f_{1}\left(x_{i}\right)=f_{2}\left(x_{i}\right)\right\} .
$$

Since $\# E \geq 2\left(N_{2}-N_{1}+m\right)-1$, without loss of generality, we assume that $\# E_{2} \geq$ $N_{2}-N_{1}+m$.

Since $f_{1}-f_{2} \neq 0, E_{2}$ is not a sampling sequence for $\left.V_{m}\right|_{\left[N_{1}, N_{2}\right]}$. We see from Proposition 2.6 that $N_{2}-N_{1} \geq 2$. We prove the conclusion with induction on $N_{2}-N_{1}$.

First, we consider the case of $N_{2}-N_{1}=2$. Since $E_{2}$ is not a sampling sequence for $\left.V_{m}\right|_{\left[N_{1}, N_{2}\right]}$, we see from Proposition 2.6 that either $E_{2} \cap\left[N_{1}, N_{1}+1\right)=\emptyset$ or $E_{2} \cap\left(N_{1}+\right.$ $\left.1, N_{2}\right]=\emptyset$. Without loss of generality, assume that $E_{2} \cap\left[N_{1}, N_{1}+1\right)=\emptyset$. 
Since $f_{1} \pm f_{2} \neq 0$, there are some $c, c^{\prime} \in \mathbb{R}^{2+m} \backslash\{0\}$ such that

$$
\left\{\begin{array}{l}
f_{1}(x)+f_{2}(x)=\sum_{n=N_{1}-m}^{N_{2}-1} c_{n} \varphi_{m}(x-n), \\
f_{1}(x)-f_{2}(x)=\sum_{n=N_{1}-m}^{N_{2}-1} c_{n}^{\prime} \varphi_{m}(x-n) .
\end{array}\right.
$$

Since \# $\left(E_{2} \cap\left[N_{1}+1, N_{2}\right]\right)=\#\left(E_{2} \cap\left[N_{1}, N_{2}\right]\right) \geq 2+m, E_{2} \cap\left[N_{1}+1, N_{2}\right]$ is a sampling sequence for $\left.V_{m}\right|_{\left[N_{1}+1, N_{2}\right]}$. We see from $f_{1}\left(x_{i}\right)=f_{2}\left(x_{i}\right)$ for $x_{i} \in E_{2}$ that $f_{1}(x)=f_{2}(x)$ on $\left[N_{1}+1, N_{2}\right]$ and

$$
c_{n}^{\prime}=0, \quad N_{2}-m-1=N_{1}-m+1 \leq n \leq N_{1}+1=N_{2}-1 .
$$

But $f_{1} \neq f_{2}$. Hence $c_{N_{1}-m}^{\prime} \neq 0$.

On the other hand, since $\#\left(E_{1} \cap\left[N_{1}, N_{1}+1\right)\right)=\#\left(E \cap\left[N_{1}, N_{1}+1\right)\right) \geq 1+m$, $E_{1} \cap\left[N_{1}, N_{1}+1\right]$ is a sampling sequence for $\left.V_{m}\right|_{\left[N_{1}, N_{1}+1\right]}$. Now we see from $f_{1}\left(x_{i}\right)=-f_{2}\left(x_{i}\right)$ for $x_{i} \in E_{1}$ that $f_{1}(x)=-f_{2}(x)$ on $\left[N_{1}, N_{1}+1\right]$ and

$$
c_{n}=0, \quad N_{1}-m \leq n \leq N_{1} .
$$

But $f_{1} \neq-f_{2}$. Hence $c_{N_{1}+1} \neq 0$. Therefore,

$$
c_{n}=c_{n}^{\prime}=0, \quad N_{1}-m+1 \leq n \leq N_{1}
$$

and

$$
c_{n} \pm c_{n}^{\prime} \neq 0, \quad n=N_{1}-m \text { or } N_{2}-1
$$

By Lemma 3.1, both $f_{1}$ and $f_{2}$ are separable. Consequently, the conclusion is true for $N_{2}-N_{1}=2$.

Now suppose that for some $N_{0} \geq 2$ and any integers $N_{1}<N_{2}$ with $N_{2}-N_{1} \leq N_{0}$ the conclusion is true. Let us consider the case of $N_{2}-N_{1}=N_{0}+1$.

Since $E_{2}$ is not a local sampling sequence for $\left.V_{m}\right|_{\left[N_{1}, N_{2}\right]}$, by Lemma 3.5, there exist integers $i_{1}, i_{1}^{\prime}, i_{2}, i_{2}^{\prime} \in\left[N_{1}, N_{2}\right]$ such that $i_{1}<i_{1}^{\prime}, i_{2}<i_{2}^{\prime},\left[i_{1}, i_{1}^{\prime}\right]$ and $\left[i_{2}, i_{2}^{\prime}\right]$ have and only have one common point, and $E_{1} \cap\left[i_{1}, i_{1}^{\prime}\right]$ and $E_{2} \cap\left[i_{2}, i_{2}^{\prime}\right]$ are sampling sequences for $\left.V_{m}\right|_{\left[i_{1}, i_{1}^{\prime}\right]}$ and $\left.V_{m}\right|_{\left[i_{2}, i_{2}^{\prime}\right]}$, respectively. Without loss of generality, we assume that $i_{1}^{\prime}=i_{2}$.

Take some $c, c^{\prime} \in \mathbb{R}^{N_{2}-N_{1}+m}$ such that (3.32) holds. Since $E_{1} \cap\left[i_{1}, i_{1}^{\prime}\right]$ is a sampling sequence for $\left[i_{1}, i_{1}^{\prime}\right]$ and $f_{1}\left(x_{i}\right)=-f_{2}\left(x_{i}\right)$ for $x_{i} \in E_{1}$, we have

$$
c_{n}=0, \quad i_{1}-m \leq n \leq i_{1}^{\prime}-1 .
$$

Similarly we get

$$
c_{n}^{\prime}=0, \quad i_{2}-m \leq n \leq i_{2}^{\prime}-1 .
$$

Hence

$$
c_{n}=c_{n}^{\prime}=0, \quad i_{1}^{\prime}-m \leq n \leq i_{1}^{\prime}-1=i_{2}-1 .
$$


Set $n_{1}=i_{2}-m-1$ and $n_{2}=i_{2}$. Then we get (3.31).

Since $f_{1} \pm f_{2} \neq 0$, neither $f_{1}$ nor $f_{2}$ is zero. Otherwise, we see from $\left|f_{1}\left(x_{i}\right)\right|=\left|f_{2}\left(x_{i}\right)\right|$ that both are zeros. Hence there exist some $k_{1}, k_{2}$ such that $c_{k_{1}}+c_{k_{1}}^{\prime} \neq 0$ and $c_{k_{2}}-c_{k_{2}}^{\prime} \neq 0$. Without loss of generality, assume that $k_{1} \leq k_{2}$.

First, we assume that $k_{1} \leq i_{2}-1 \leq k_{2}$. Then we have $k_{1} \leq i_{1}^{\prime}-m-1$ and $k_{2} \geq i_{1}^{\prime}=i_{2}$.

Note that $f_{1}=\sum_{n=N_{1}-m}^{N_{2}-1} \frac{c_{n}+c_{n}^{\prime}}{2} \varphi_{m}(\cdot-n)$. We have $\left.f_{1}\right|_{\left[N_{1}, i_{1}^{\prime}\right]} \neq 0$. Since $E \cap\left[N_{1}, i_{1}^{\prime}\right]$ is a sampling sequence for $\left.V_{m}\right|_{\left[N_{1}, i_{1}^{\prime}\right]}$, we see from (3.30) that $\left.f_{2}\right|_{\left[N_{1}, i_{1}^{\prime}\right]} \neq 0$. Similarly we can prove that $\left.f_{i}\right|_{\left[i_{2}, N_{2}\right]} \neq 0, i=1,2$. Hence we can find integers $j_{l}^{(i)}, 1 \leq i, l \leq 2$, as desired.

Next we assume that for any $k_{1}, k_{2}$ with $c_{k_{1}}+c_{k_{1}}^{\prime} \neq 0$ and $c_{k_{2}}-c_{k_{2}}^{\prime} \neq 0, k_{1}$ and $k_{2}$ lie on the same side of $i_{2}-1$. Without loss of generality, we assume that $k_{1}, k_{2} \leq i_{2}-1$. Then we have

$$
c_{n}=c_{n}^{\prime}=0, \quad n \geq i_{2}-m
$$

Hence

$$
\left\{\begin{array}{l}
f_{1}(x)+f_{2}(x)=\sum_{n=N_{1}-m}^{i_{2}-m-1} c_{n} \varphi_{m}(x-n), \\
f_{1}(x)-f_{2}(x)=\sum_{n=N_{1}-m}^{i_{2}-m-1} c_{n}^{\prime} \varphi_{m}(x-n) .
\end{array}\right.
$$

Take $m+1$ points $y_{1}, \ldots, y_{m+1}$ in $\left(i_{2}-1, i_{2}\right) \backslash E$ and set $\tilde{E}=\left(E \cap\left[N_{1}, i_{2}\right]\right) \cup\left\{y_{i}: 1 \leq\right.$ $i \leq m+1\}$. Then $\tilde{E}$ meets (1.1) - (1.4) if $\left(E, N_{2}\right)$ is replaced by $\left(\tilde{E}, i_{2}\right)$.

Let $\tilde{E}_{1}=\left(E_{1} \cap\left[N_{1}, i_{2}\right]\right) \cup\left\{y_{i}: 1 \leq i \leq m+1\right\}$ and $\tilde{E}_{2}=E_{2} \cap\left[N_{1}, i_{2}\right]$. Then we have $\tilde{E}=\tilde{E}_{1} \cup \tilde{E}_{2}$ and $f_{1}\left(x_{i}\right)=f_{2}\left(x_{i}\right)$ for $x_{i} \in \tilde{E}_{2}$.

On the other hand, since $E_{1} \cap\left[i_{1}, i_{1}^{\prime}\right]$ is a sampling sequence for $\left.V_{m}\right|_{\left[i_{1}, i_{1}^{\prime}\right]}$ and $\left(f_{1}+\right.$ $\left.f_{2}\right)\left.\right|_{E_{1}}=0$, we have $\left.\left(f_{1}+f_{2}\right)\right|_{\left[i_{1}, i_{1}^{\prime}\right]}=0$. Hence $f_{1}\left(y_{i}\right)+f_{2}\left(y_{i}\right)=0,1 \leq i \leq m+1$. Therefore $\left.\left(f_{1}+f_{2}\right)\right|_{\tilde{E}_{1}}=0$. Since $i_{2}-N_{1} \leq N_{2}-N_{1}-1$, we see from the inductive assumption that we can find integers $j_{l}^{(i)}, 1 \leq i, l \leq 2$, as desired.

Finally, we prove that $\left|f_{1}(x)\right|=\left|f_{2}(x)\right|$. We see from the above arguments that there exist integers $i_{1}<i_{1}^{\prime}=i_{2}<i_{2}^{\prime}$ such that $E_{l} \cap\left[i_{l}, i_{l}^{\prime}\right]$ is a sampling sequence for $\left.V_{m}\right|_{\left[i_{l}, i_{l}^{\prime}\right]}$, $l=1,2$. Hence $f_{1}=-f_{2}$ on $\left[i_{1}, i_{1}^{\prime}\right]$ and $f_{1}=f_{2}$ on $\left[i_{2}, i_{2}^{\prime}\right]$. Take some $F_{l} \subset\left[i_{l}, i_{l}^{\prime}\right]$ such that $\# F_{l}=2 m+3$. Then $F_{1} \cup E \cap\left[N_{1}, i_{1}^{\prime}\right]$ and $F_{2} \cup E \cap\left[i_{2}, N_{2}\right]$ are phaseless sampling sequences for $\left.V_{m}\right|_{\left[N_{1}, i_{1}^{\prime}\right]}$ and $\left.V_{m}\right|_{\left[i_{2}, N_{2}\right]}$, respectively. Since $i_{1}^{\prime}-N_{1}<N_{2}-N_{1}$ and $N_{2}-i_{2}<N_{2}-N_{1}$, we see from the inductive assumption that $\left|f_{1}(x)\right|=\left|f_{2}(x)\right|$ on $\left[N_{1}, N_{2}\right]$.

By induction, the conclusion is true for any $N_{1}<N_{2}$. This completes the proof.

\section{Global Phaseless Sampling in Spline Spaces}

In this section, we give a proof for Theorem 1.3. First, we present some equivalent characterization for phaseless sampling sequences, for which we leave the proof to interested readers.

Lemma 4.1 Suppose that $m \geq 2$. For a sequence E satisfying (P1) in Theorem 1.3, (P2) is equivalent to any one of the followings, 
(i). For any $n_{0} \in \mathbb{Z}$,

$$
\lim _{n \rightarrow \infty}\left(\#\left(E \cap\left[n_{0}, n\right]\right)-2\left(n-n_{0}\right)\right)=\lim _{n \rightarrow-\infty}\left(\#\left(E \cap\left[n, n_{0}\right]\right)-2\left(n_{0}-n\right)\right)=\infty .
$$

(ii). For any $n_{0} \in \mathbb{Z}$,

$$
\begin{aligned}
\sup _{n>n_{0}} & \left(\#\left(E \cap\left[n_{0}, n\right]\right)-2\left(n-n_{0}\right)\right) \\
& =\sup _{n<n_{0}}\left(\#\left(E \cap\left[n, n_{0}\right]\right)-2\left(n_{0}-n\right)\right)=\infty .
\end{aligned}
$$

Proof of Theorem 1.3. Necessity. First, we show that $\#(E \cap(n, n+1)) \geq 1$ for any $n \in \mathbb{Z}$. Assume on the contrary that $\#(E \cap(n, n+1))=0$ for some $n \in \mathbb{Z}$. Let

$$
\begin{aligned}
& f_{1}=\sum_{k \geq n} \varphi_{m}(\cdot-k)+\sum_{k \leq n-m} \varphi_{m}(\cdot-k), \\
& f_{2}=\sum_{k \geq n} \varphi_{m}(\cdot-k)-\sum_{k \leq n-m} \varphi_{m}(\cdot-k),
\end{aligned}
$$

Then we have

$$
\begin{aligned}
& f_{1}(x)=-f_{2}(x), \quad x \leq n, \\
& f_{1}(x)=f_{2}(x), \quad x \geq n+1 .
\end{aligned}
$$

Hence $\left|f_{1}(x)\right|=\left|f_{2}(x)\right|$ for $x \in E$. But $f_{1} \pm f_{2} \neq 0$ and neither $f_{1}$ nor $f_{2}$ is separable, which contradicts with the hypothesis.

Now assume that $\#\left(E \cap\left(i_{1}, i_{2}\right)\right) \leq 2\left(i_{2}-i_{1}\right)-2$ for some $i_{1}<i_{2}-1$. Let

$$
\begin{aligned}
& n_{1}=\max \left\{n<i_{2}: \#\left(E \cap\left(n, i_{2}\right)\right) \leq 2\left(i_{2}-n\right)-2\right\}, \\
& n_{2}=\min \left\{n>n_{1}: \#\left(E \cap\left(n_{1}, n\right)\right) \leq 2\left(n-n_{1}\right)-2\right\} .
\end{aligned}
$$

Then we have $i_{1} \leq n_{1}<n_{2} \leq i_{2}$ and (3.12) - (3.16) hold. Hence there is some $E_{2} \subset E$ such that

$$
\begin{aligned}
& E \cap\left(\left(-\infty, n_{1}\right] \cup\left[n_{2}, \infty\right)\right) \subset E_{2}, \\
& E_{2} \cap(n, n+1)=1, \quad n_{1} \leq n \leq n_{2}-2, \\
& E_{2} \cap\left(n_{1}, n_{2}\right)=n_{2}-n_{1}-1, \quad n_{1} \leq n \leq n_{2}-2 .
\end{aligned}
$$

Let $E_{1}=E \backslash E_{2}$ and

$$
\begin{aligned}
& A_{1}=\left[\varphi_{m}\left(x_{i}-n\right)\right]_{x_{i} \in E_{1}, n_{1} \leq n \leq n_{2}-1}, \\
& A_{2}=\left[\varphi_{m}\left(x_{i}-n\right)\right]_{x_{i} \in E_{2} \cap\left(n_{1}, n_{2}\right), n_{1}-m \leq n \leq n_{2}-m-1} .
\end{aligned}
$$

Since $\# E_{1}=n_{2}-n_{1}-1$, we see from Proposition 3.3 that there is some $c \in \mathbb{R}^{n_{2}-n_{1}}$, none of whose entries is zero, such that

$$
A_{1} c=0 \text {. }
$$


Similarly, there is some $c^{\prime} \in \mathbb{R}^{n_{2}-n_{1}}$, none of whose entries is zero, such that

$$
A_{2} c^{\prime}=0
$$

Again, by multiplying a factor, we can assume that $c_{i} \pm c_{i}^{\prime} \neq 0, n_{1} \leq i \leq n_{2}-m-1$. Let

$$
\begin{aligned}
f_{1} & =\sum_{n=n_{1}}^{n_{2}-1} c_{n} \varphi_{m}(\cdot-n)+\sum_{n=n_{1}-m}^{n_{2}-m-1} c_{n}^{\prime} \varphi_{m}(\cdot-n), \\
f_{2} & =\sum_{n=n_{1}}^{n_{2}-1} c_{n} \varphi_{m}(\cdot-n)-\sum_{n=n_{1}-m}^{n_{2}-m-1} c_{n}^{\prime} \varphi_{m}(\cdot-n) .
\end{aligned}
$$

If $n_{1} \leq n_{2}-m-1$, then both $f_{1}$ and $f_{2}$ are nonseparable. If $n_{1}>n_{2}-m-1$, then $n_{1}-\left(n_{2}-m-1\right) \leq m-1$. Again, both $f_{1}$ and $f_{2}$ are nonseparable. Moreover,

$$
f_{1}(x)= \begin{cases}-f_{2}(x), & \text { if } x \leq n_{1} \text { or } x \in E_{1}=E_{1} \cap\left(n_{1}, n_{2}\right) \\ f_{2}(x), & \text { if } x \geq n_{2} \text { or } x \in E_{2} \cap\left(n_{1}, n_{2}\right) .\end{cases}
$$

Since $f_{1} \pm f_{2} \neq 0$, it is impossible to recover $f_{1}$ or $f_{2}$, which contradicts with the hypothesis. Hence (P1) is true.

Next we prove (P2) for $m \geq 2$. Assume that for some $n_{0}$ and any $n_{2}>n_{1} \geq n_{0}$, $\#\left(E \cap\left[n_{1}, n_{2}\right]\right) \leq 2\left(n_{2}-n_{1}+m\right)-2$. Then we have

$$
K:=\sup _{n>n_{0}} \#\left(E \cap\left[n_{0}, n\right]\right)-2\left(n-n_{0}\right)<\infty .
$$

Otherwise, there is some $n>n_{0}$ such that \# $\left(E \cap\left[n_{0}, n\right]\right)-2\left(n-n_{0}\right)>4 m$. Hence either $\#\left(E \cap\left[n_{0}, n_{0}+1\right]\right) \geq 2 m+1$ or $\#\left(E \cap\left[n_{0}+1, n\right]\right) \geq 2\left(n-n_{0}-1\right)+2 m-1$, which contradicts with the assumption.

It follows that there exists some $n_{2}>n_{0}$ such that

$$
K=\#\left(E \cap\left[n_{0}, n_{2}\right]\right)-2\left(n_{2}-n_{0}\right) .
$$

For $n>n_{2}$, we have

$$
\#\left(E \cap\left(n_{2}, n\right]\right) \leq 2\left(n-n_{2}\right) .
$$

Moreover,

$$
\#(E \cap(n, n+1]) \leq 3, \quad n \geq n_{2} .
$$

Otherwise, we have

$$
\#\left(E \cap\left(n_{2}, n+1\right]\right)=\#\left(E \cap\left(n_{2}, n\right]\right)+\#(E \cap(n, n+1]) \geq 2\left(n-n_{2}\right)+3 .
$$

Hence

$$
\#\left(E \cap\left[n_{0}, n+1\right]\right)-2\left(n+1-n_{0}\right) \geq K+1,
$$

which is impossible.

Let $a_{i}=\#(E \cap(i-1, i]), i \geq n_{2}+1$. Then we have $1 \leq a_{i} \leq 3$. There are three cases. 
(i). There are infinitely many $i>n_{2}$ such that $a_{i}=3$.

Suppose that $a_{i_{k}}=3$ for $k \geq 1$ and $a_{i} \leq 2$ for $i \geq n_{2}+1$ and $i \notin\left\{i_{k}: k \geq 1\right\}$. Set $i_{0}=n_{2}$. We conclude that $i_{k}-i_{k-1} \geq 2$ for $k \geq 1$.

In fact, if $i_{1}=n_{2}+1$, then we have $\#\left(E \cap\left[n_{0}, i_{1}\right]\right)-2\left(i_{1}-n_{0}\right)=K+1$, which is impossible. On the other hand, if $i_{k}=i_{k-1}+1$ for some $k \geq 2$, then

$$
\begin{aligned}
\#\left(E \cap\left[n_{0}, i_{k}\right]\right)= & \#\left(E \cap\left[n_{0}, n_{2}\right]\right)+\#\left(E \cap\left(n_{2}, i_{k-1}-1\right]\right) \\
& +\#\left(E \cap\left(i_{k-1}-1, i_{k}\right]\right) \\
\geq & K+2\left(n_{2}-n_{0}\right)+2\left(i_{k-1}-1-n_{2}\right)-1+6 \\
= & K+2\left(i_{k}-n_{0}\right)+1 .
\end{aligned}
$$

Again, we get a contradiction.

Observe that

$$
\begin{aligned}
\#\left(E \cap\left(i_{k-1}, i_{k}\right]\right) & =\#\left(E \cap\left(i_{k-1}, i_{k}-1\right]\right)+\#\left(E \cap\left(i_{k}-1, i_{k}\right]\right) \\
& \geq 2\left(i_{k}-i_{k-1}\right) .
\end{aligned}
$$

It is easy to check by induction that for $k \geq 1$,

$$
\#\left(E \cap\left(i_{k-1}, i_{k}\right]\right)=2\left(i_{k}-i_{k-1}\right) .
$$

(ii). There are only finitely many $i>n_{2}$ such that $a_{i}=3$.

Suppose that $a_{i_{1}}, \ldots, a_{i_{r}}=3$. Let $i_{0}=n_{2}$ and $i_{k}=i_{r}+2(k-r)$ for $k>r$. Similarly we can show that (4.2) is true for $1 \leq k \leq r$. Hence

$$
2\left(i_{k}-i_{k-1}\right)-1 \leq \#\left(E \cap\left(i_{k-1}, i_{k}\right]\right) \leq 2\left(i_{k}-i_{k-1}\right), \quad k \geq 1 .
$$

(iii). $a_{i} \leq 2$ for $i>n_{2}$.

In this case, set $i_{k}=n_{2}+2 k, k \geq 0$. Then (4.3) is true.

In all three cases, we get $n_{2}=i_{0}<i_{1}<\ldots<i_{k}<\ldots$ such that $i_{k}-i_{k-1} \geq 2$ and (4.3) is true.

Take some $E_{2} \subset E$ such that

$$
\begin{aligned}
& \#\left(E_{2} \cap(n, n+1)\right) \geq 1, \quad n \in \mathbb{Z}, \\
& \#\left(E_{2} \cap\left(i_{0}, i_{1}\right]\right)=\#\left(E_{2} \cap\left[i_{0}, i_{1}\right]\right)=i_{1}-i_{0}+1, \\
& \#\left(E_{2} \cap\left(i_{k-1}, i_{k}\right]\right)=i_{k}-i_{k-1}, \quad k \geq 2, \\
& \#\left(E_{2} \cap\left[n, i_{0}\right)\right)=i_{0}-n, \quad n<i_{0}, \\
& E \cap\left(i_{0}, i_{0}+1\right] \subset E_{2} .
\end{aligned}
$$

Let $E_{1}=E \backslash E_{2}$. Then

$$
\begin{aligned}
& \#\left(E_{1} \cap\left(i_{0}, i_{0}+1\right]\right)=0, \\
& \#\left(E_{1} \cap\left(i_{0}, i_{1}\right]\right) \leq i_{1}-i_{0}-1, \\
& \#\left(E_{1} \cap\left(i_{k-1}, i_{k}\right]\right) \leq i_{k}-i_{k-1}, \quad k \geq 2 .
\end{aligned}
$$


Since $\#\left(E_{2} \cap\left[i_{0}, i_{1}\right]\right)=i_{1}-i_{0}+1<i_{1}-i_{0}+m$, there exist some $c_{i_{0}-m}^{\prime}, \ldots, c_{i_{1}-1}^{\prime} \in \mathbb{R}$, not all of which are zeros, such that

$$
h_{2}\left(x_{i}\right)=\sum_{n=i_{0}-m}^{i_{1}-1} c_{n}^{\prime} \varphi_{m}\left(x_{i}-n\right)=0, \quad x_{i} \in E_{2} \cap\left[i_{0}, i_{1}\right] .
$$

Consider the linear system

$$
h_{2}\left(x_{i}\right)=\sum_{n=i_{0}-m}^{i_{2}-1} c_{n}^{\prime} \varphi_{m}\left(x_{i}-n\right)=0, \quad x_{i} \in E_{2} \cap\left(i_{1}, i_{2}\right],
$$

where $c_{n}^{\prime}, i_{1} \leq n \leq i_{2}-1$ are considered as unknowns. Denote $E_{2} \cap\left(i_{1}, i_{2}\right]=\left\{y_{i}: 1 \leq i \leq\right.$ $\left.i_{2}-i_{1}\right\}$. Then the above system can be rewritten as

$$
\left(\begin{array}{cccc}
* & 0 & \ldots & 0 \\
* & * & \ldots & 0 \\
& \ldots & \ldots & 0 \\
? & ? & \ldots & *
\end{array}\right)\left(\begin{array}{c}
c_{i_{1}}^{\prime} \\
\vdots \\
c_{i_{2}-1}^{\prime}
\end{array}\right)=\left(\begin{array}{c}
b_{1} \\
\vdots \\
b_{i_{2}-i_{1}}
\end{array}\right)
$$

where $*$ stands for non-zero entries and $b_{i}=-\sum_{n=i_{0}-m}^{i_{1}-1} c_{n}^{\prime} \varphi_{m}\left(y_{i}-n\right), 1 \leq i \leq i_{1}-n_{2}$. Hence there is a unique solution to the linear system. Consequently, for $x_{i} \in E_{2} \cap\left(i_{1}, i_{2}\right]$,

$$
h_{2}\left(x_{i}\right)=\sum_{n=i_{0}-m}^{i_{2}-1} c_{n}^{\prime} \varphi_{m}\left(x_{i}-n\right)=0 .
$$

Note that the above identity is also true for $x_{i} \in E_{2} \cap\left[i_{0}, i_{1}\right]$ since $\varphi_{m}\left(x_{i}-n\right)=0$ for $x_{i} \leq i_{1} \leq n$. By induction, it is easy to see that there exists a sequence of real numbers $\left\{c_{n}^{\prime}: n \in \mathbb{Z}\right\}$ such that

$$
h_{2}\left(x_{i}\right)=\sum_{n \in \mathbb{Z}} c_{n}^{\prime} \varphi_{m}\left(x_{i}-n\right)=0, \quad x_{i} \in E_{2} .
$$

On the other hand, by (4.5) and (4.6), there exist some $c_{i_{0}}, \ldots, c_{i_{1}-1} \in \mathbb{R}$, not all of which are zeros, such that

$$
h_{1}\left(x_{i}\right)=\sum_{n=i_{0}}^{i_{1}-1} c_{n} \varphi_{m}\left(x_{i}-n\right)=0, \quad x_{i} \in E_{1} \cap\left(i_{0}, i_{1}\right] .
$$

Put $c_{n}=0$ for $n<i_{0}$. We get

$$
h_{1}\left(x_{i}\right)=\sum_{n \leq i_{1}-1} c_{n} \varphi_{m}\left(x_{i}-n\right)=0, \quad x_{i} \in E_{1} \cap\left(-\infty, i_{1}\right] .
$$

Since \# $\left(E_{1} \cap\left(i_{k-1}, i_{k}\right]\right) \leq i_{k}-i_{k-1}, k \geq 2$, similarly we can find some $c_{n} \in \mathbb{R}$ for $n \geq i_{1}$, which might not be unique, such that

$$
h_{1}\left(x_{i}\right)=\sum_{n \in \mathbb{Z}} c_{n} \varphi_{m}\left(x_{i}-n\right)=0, \quad x_{i} \in E_{1} .
$$


For any $n \in \mathbb{Z}$ with $c_{n}^{\prime} \neq 0$, there are at most two numbers $r$ such that $c_{n}+r c_{n}^{\prime}=0$ or $c_{n}-r c_{n}^{\prime}=0$. Hence there is some $r \in[1,2]$ such that $c_{n} \pm r c_{n}^{\prime} \neq 0$ if $c_{n}^{\prime} \neq 0$. Set

$$
\begin{aligned}
f_{1} & =\sum_{n \in \mathbb{Z}} \frac{c_{n}+r c_{n}^{\prime}}{2} \varphi_{m}(\cdot-n), \\
f_{2} & =\sum_{n \in \mathbb{Z}} \frac{c_{n}-r c_{n}^{\prime}}{2} \varphi_{m}(\cdot-n) .
\end{aligned}
$$

By (4.4), there is not an integer $n$ such that $c_{n}^{\prime}=\ldots=c_{n+m-1}^{\prime}=0$. Otherwise, $c_{n}^{\prime}=0$ for all $n \in \mathbb{Z}$. The same is true for $c_{n} \pm r c_{n}^{\prime}$. Hence both $f_{1}$ and $f_{2}$ are nonseparable. Since $f_{1} \pm f_{2} \neq 0$ and $\left|f_{1}\left(x_{i}\right)\right|=\left|f_{2}\left(x_{i}\right)\right|$ for $x_{i} \in E$, we can not recover $f_{1}$ up to a sign, which contradicts with the hypothesis. Similarly we can show that for any integer $n_{0}$, there exist integers $i_{1}<i_{2} \leq n_{0}$ such that \# $\left(E \cap\left[i_{1}, i_{2}\right]\right) \geq 2\left(i_{2}-i_{1}+m\right)-1$. Hence (P2) is true.

It remains to prove (P2') for $m=1$. By (P1), there is some $E_{2}=\left\{x_{i}: i \in \mathbb{Z}\right\} \subset E$ such that $x_{i} \in(i, i+1)$. Let $E_{1}=E \backslash E_{2}$. Then $E_{1} \neq \emptyset$.

Take some $c_{-1}^{\prime}, c_{0}^{\prime} \in \mathbb{R} \backslash\{0\}$ such that

$$
c_{-1}^{\prime} \varphi_{1}\left(x_{0}+1\right)+c_{0}^{\prime} \varphi_{1}\left(x_{0}\right)=0 .
$$

By induction, for any $n \in \mathbb{Z}$, we can find $c_{n}^{\prime} \in \mathbb{R}$ successively such that

$$
c_{n-1}^{\prime} \varphi_{1}\left(x_{n}-n+1\right)+c_{n}^{\prime} \varphi_{1}\left(x_{n}-n\right)=0 .
$$

It is easy to see that $c_{n}^{\prime} \neq 0, n \in \mathbb{Z}$. Set $h_{2}(x)=\sum_{n \in \mathbb{Z}} c_{n}^{\prime} \varphi_{m}(x-n)$. Then $\left.h_{2}\right|_{E_{2}}=0$.

Denote $b_{n}=\#(E \cap[n-1, n]), n \in \mathbb{Z}$. First, we show that there exists some $n \in \mathbb{Z}$ such that $b_{n} \geq 3$.

Assume on the contrary that $b_{n} \leq 2, n \in \mathbb{Z}$. Then $E_{1} \cap[n, n+1] \leq 1$ for any $n \in \mathbb{Z}$. Since $\#(E \cap(0,3)) \geq 5$ and $\#\left(E_{2} \cap(0,3)\right)=3$, we have $\#\left(E_{1} \cap(0,3)\right) \geq 2$. Moreover, since $b_{n} \leq 2,1$ or 2 is not in $E_{1}$. Consequently, there is some $y_{0} \in E_{1} \cap(0,3) \backslash \mathbb{Z}$. Since $\#\left(E_{1} \cap[n, n+1]\right) \leq 1$, it is easy to see that there is some $h_{1} \in V_{1}$ such that $h_{1} \neq 0$ and $\left.h_{1}\right|_{E_{1}}=0$. Since $h_{2}$ is nonseparable and $h_{1}, h_{2} \neq 0$, similarly to the proof of (P2) we get a contradiction.

Take some $n_{0} \in \mathbb{Z}$ such that $b_{n_{0}} \geq 3$. Denote $\left\{n: b_{n} \geq 3\right\}$ by $\left\{n_{k}: k_{1} \leq k \leq k_{2}\right\}$.

Suppose that $k_{2}<\infty$. Then $b_{n} \leq 2$ for any $n>n_{k_{2}}$. If there is some $i_{0} \in E \cap\left[n_{k_{2}}, \infty\right) \cap$ $\mathbb{Z}$, it follows from $b_{i_{0}+1} \leq 2$ that $\#\left(E \cap\left(i_{0}, i_{0}+1\right]\right)=1$. Hence $E_{1} \cap\left(i_{0}, i_{0}+1\right]=\emptyset$. Since $\#\left(E \cap\left(i_{0}, i_{0}+2\right)\right) \geq 3$, there is some $y_{0} \in\left(i_{0}+1, i_{0}+2\right) \cap E_{1}$. Hence there exist constants $c_{n}, n \geq i_{0}$, not all of which are zeros, such that

$$
h_{1}(x):=\sum_{n \geq i_{0}} c_{n} \varphi_{1}(x-n)=0
$$

holds for $x \in E_{1} \cap\left(i_{0}+1, \infty\right)$ and therefore for all $x \in E_{1}$. Similarly to the above we get a contradiction. Hence $E \cap\left[n_{k_{2}}, \infty\right) \cap \mathbb{Z}=\emptyset$.

If $b_{n}=1$ for some $n>n_{k_{2}}$, with similar arguments we get a contradiction. Hence

$$
\#(E \cap(n, n+1))=2, \quad n \geq n_{k_{2}} .
$$


Similar arguments show that $\#\left(E \cap(n-1, n)=2\right.$ for $n \leq n_{k_{1}}$.

Sufficiency. Let $f_{1}, f_{2} \in V_{m}$ be such that $\left|f_{1}\left(x_{i}\right)\right|=\left|f_{2}\left(x_{i}\right)\right|$ for $x_{i} \in E$. Assume that $f_{1} \pm f_{2} \neq 0$. Then neither $f_{1}$ nor $f_{2}$ is equal to zero. In fact, we see from (P1) that $E \cap\left[n_{1}, n_{2}\right]$ is a sampling sequence for $\left.V_{m}\right|_{\left[n_{1}, n_{2}\right]}$ whenever $n_{2}-n_{1} \geq m+1$. Since $\left|f_{1}(x)\right|=\left|f_{2}(x)\right|$ on $E$, if $f_{1}=0$, then we have $f_{2}=0$. Therefore, $f_{1} \pm f_{2}=0$, which is a contradiction.

First, we consider the case of $m \geq 2$. Suppose that a sequence $E$ satisfies (P1) and (P2). Define

$$
E_{1}=\left\{x_{i}: f_{1}\left(x_{i}\right)=-f_{2}\left(x_{i}\right)\right\} \quad \text { and } \quad E_{2}=\left\{x_{i}: f_{1}\left(x_{i}\right)=f_{2}\left(x_{i}\right)\right\} .
$$

By (P2), there exists integers $n_{i}$ and $n_{i}^{\prime}, i \in \mathbb{Z}$, such that

$$
n_{i}<n_{i}^{\prime} \leq n_{i+1}<n_{i+1}^{\prime}, \quad i \in \mathbb{Z}
$$

and

$$
\#\left(E \cap\left[n_{i}, n_{i}^{\prime}\right]\right) \geq 2\left(n_{i}^{\prime}-n_{i}+m\right)-1 .
$$

Hence for any $i \in \mathbb{Z}$, either $\#\left(E_{1} \cap\left[n_{i}, n_{i}^{\prime}\right]\right) \geq n_{i}^{\prime}-n_{i}+m$ or $\#\left(E_{2} \cap\left[n_{i}, n_{i}^{\prime}\right]\right) \geq n_{i}^{\prime}-n_{i}+m$. There are two case.

(i) There exist some $i \neq j$ such that $\#\left(E_{1} \cap\left[n_{i}, n_{i}^{\prime}\right]\right) \geq n_{i}^{\prime}-n_{i}+m$ and $\#\left(E_{2} \cap\left[n_{j}, n_{j}^{\prime}\right]\right) \geq$ $n_{j}^{\prime}-n_{j}+m$.

Without loss of generality, we assume that $i<j$. We see from Lemma 3.4 that there exist integers $i_{1}<i_{1}^{\prime}=i_{2}<i_{2}^{\prime}$ such that $E_{l} \cap\left[i_{l}, i_{l}^{\prime}\right]$ is a sampling sequence for $\left.V_{m}\right|_{\left[i_{l}, i_{l}^{\prime}\right]}$, $l=1,2$.

(ii) For $l=1$ or $2, \#\left(E_{l} \cap\left[n_{i}, n_{i}^{\prime}\right]\right) \geq n_{i}^{\prime}-n_{i}+m$ for all $i \in \mathbb{Z}$.

Without loss of generality, assume that $\#\left(E_{2} \cap\left[n_{i}, n_{i}^{\prime}\right]\right) \geq n_{i}^{\prime}-n_{i}+m$ for all $i \in \mathbb{Z}$. By Lemma 3.2, there exists $\left[\tilde{n}_{i}, \tilde{n}_{i}^{\prime}\right] \subset\left[n_{i}, n_{i}^{\prime}\right]$ such that $E_{2} \cap\left[\tilde{n}_{i}, \tilde{n}_{i}^{\prime}\right]$ is a sampling sequence for $\left.V_{m}\right|_{\left[\tilde{n}_{i}, \tilde{n}_{i}^{\prime}\right]}, i \in \mathbb{Z}$.

If $E_{2} \cap\left[\tilde{n}_{i}, \tilde{n}_{j}^{\prime}\right]$ is a sampling sequence for $\left.V_{m}\right|_{\left[\tilde{n}_{i}, \tilde{n}_{j}^{\prime}\right]}$ for any $i<j$, then we have $f_{1}-f_{2}=$ 0 on $\left[\tilde{n}_{i}, \tilde{n}_{j}^{\prime}\right]$ for any $i<j$. Hence $f_{1}=f_{2}$, which contradicts with the assumption. Consequently, there exist some $i<j$ such that $E_{2} \cap\left[\tilde{n}_{i}, \tilde{n}_{j}^{\prime}\right]$ is not a sampling sequence for $\left.V_{m}\right|_{\left[\tilde{n}_{i}, \tilde{n}_{j}^{\prime}\right]}$. By Proposition 2.6, there exist integers $l, l^{\prime}$ with $\tilde{n}_{i}^{\prime} \leq l<l^{\prime} \leq \tilde{n}_{j}$ such that $\#\left(E_{2} \cap\left(l, l^{\prime}\right)\right) \leq l^{\prime}-l-m-1$. Since $\#\left(E \cap\left(l, l^{\prime}\right)\right) \geq 2\left(l^{\prime}-l\right)-1$, we have $\#\left(E_{1} \cap\left(l, l^{\prime}\right)\right) \geq$ $l^{\prime}-l+m$.

Using Lemma 3.4 again, we get integers $i_{1}<i_{1}^{\prime}=i_{2}<i_{2}^{\prime}$ such that $E_{l} \cap\left[i_{l}, i_{l}^{\prime}\right]$ is a sampling sequence for $\left.V_{m}\right|_{\left[i_{l}, i_{l}^{\prime}\right]}, l=1,2$.

Now assume that

$$
\begin{aligned}
& f_{1}+f_{2}=\sum_{n \in \mathbb{Z}} c_{n} \varphi_{m}(\cdot-k), \\
& f_{1}-f_{2}=\sum_{n \in \mathbb{Z}} c_{n}^{\prime} \varphi_{m}(\cdot-k) .
\end{aligned}
$$

Since $E_{l} \cap\left[i_{l}, i_{l}^{\prime}\right]$ is a sampling sequence for $\left.V_{m}\right|_{\left[i_{l}, i_{l}^{\prime}\right]}, l=1,2$, we have

$$
c_{i}=0, \quad i_{1}-m \leq i \leq i_{1}^{\prime}-1,
$$




$$
c_{i}^{\prime}=0, \quad i_{2}-m \leq i \leq i_{2}^{\prime}-1 .
$$

Hence

$$
c_{i} \pm c_{i}^{\prime}=0, \quad i_{2}-m \leq i \leq i_{2}-1 .
$$

On the other hand, since $f_{1}, f_{2} \neq 0$, there exist some $k_{1}, k_{2}$ such that $c_{k_{1}}+c_{k_{1}}^{\prime} \neq 0$ and $c_{k_{2}}-c_{k_{2}}^{\prime} \neq 0$. If $k_{1}$ and $k_{2}$ lie on the two sides of $i_{2}-1$, respectively, then similar arguments as that in the proof of Lemma 3.6 show that both $f_{1}$ and $f_{2}$ are separable.

Next we assume that both $k_{1}$ and $k_{2}$ are on the same side of $i_{2}-1$ whenever $c_{k_{1}}+c_{k_{1}}^{\prime} \neq 0$ and $c_{k_{2}}-c_{k_{2}}^{\prime} \neq 0$. Without loss of generality, we assume that $c_{i} \pm c_{i}^{\prime}=0$ for $i>i_{2}-1$ and $c_{k_{0}}+c_{k_{0}}^{\prime} \neq 0$, where $k_{0}=\max \left\{i:\left|c_{i}+c_{i}^{\prime}\right|+\left|c_{i}-c_{i}^{\prime}\right|>0\right\}$. In this case,

$$
\begin{aligned}
f_{1}+f_{2} & =\sum_{n \leq k_{0}} c_{n} \varphi_{m}(\cdot-k), \\
f_{1}-f_{2} & =\sum_{n \leq k_{0}} c_{n}^{\prime} \varphi_{m}(\cdot-k) .
\end{aligned}
$$

Hence $f_{1}(x)=f_{2}(x)=0$ for $x \geq k_{0}+m+1$.

Take some $\left\{y_{i}: 1 \leq i \leq 4 m\right\} \subset\left[k_{0}+m+1, k_{0}+m+2\right]$. Let $\tilde{E}_{1}=\left(E_{1} \cap\left(-\infty, k_{0}+m+\right.\right.$ 1)) $\cup\left\{y_{i}: 1 \leq i \leq 2 m\right\}, \tilde{E}_{2}=\left(E_{2} \cap\left(-\infty, k_{0}+m+1\right)\right) \cup\left\{y_{i}: 2 m+1 \leq i \leq 4 m\right\}$. Then we have $\left.\left(f_{1}+f_{2}\right)\right|_{\tilde{E}_{1}}=0$ and $\left.\left(f_{1}-f_{2}\right)\right|_{\tilde{E}_{2}}=0$. Moreover, $\tilde{E}_{l} \cap\left[k_{0}+m+1, k_{0}+m+2\right]$ is a sampling sequence for $\left.V_{m}\right|_{\left[k_{0}+m+1, k_{0}+m+2\right]}, l=1,2$.

Since \# $\left(\tilde{E}_{l} \cap\left(k_{0}+m, k_{0}+m+1\right)\right) \geq 1$ for $l=1$ or 2 , one of $\tilde{E}_{l} \cap\left[k_{0}+m, k_{0}+m+2\right]$, $l=1,2$, is a sampling sequence for $\left.V_{m}\right|_{\left[k_{0}+m, k_{0}+m+2\right]}$. Without loss of generality, assume that $\tilde{E}_{2} \cap\left[k_{0}+m, k_{0}+m+2\right]$ is a sampling sequence for $\left.V_{m}\right|_{\left[k_{0}+m, k_{0}+m+2\right]}$. Then we have $c_{k_{0}}^{\prime}=0$.

Repeating the previous arguments we get a sequence of integers

$$
\ldots \leq \tilde{n}_{i}<\tilde{n}_{i}^{\prime} \leq \ldots \leq \tilde{n}_{0}<\tilde{n}_{0}^{\prime} \leq k_{0}+m
$$

such that for each $i \leq 0$, one of $E_{l} \cap\left[\tilde{n}_{i}, \tilde{n}_{i}^{\prime}\right], l=1,2$, is a sampling sequence for $\left.V_{m}\right|_{\left[\tilde{n}_{i}, \tilde{n}_{i}^{\prime}\right]}$. We conclude that there exist some $l<l^{\prime} \leq k_{0}+m$ such that $E_{1} \cap\left[l, l^{\prime}\right]$ is a sampling sequence for $\left.V_{m}\right|_{\left[l, l^{\prime}\right]}$.

Assume on the contrary that $E_{1} \cap\left[l, l^{\prime}\right]$ is not a sampling sequence for $\left.V_{m}\right|_{\left[l, l^{\prime}\right]}$ for any $l<l^{\prime} \leq k_{0}+m$. Then $E_{2} \cap\left[\tilde{n}_{i}, \tilde{n}_{i}^{\prime}\right]$ is a sampling sequence for $\left.V_{m}\right|_{\left[\tilde{n}_{i}, \tilde{n}_{i}^{\prime}\right]}$ for all $i \leq 0$. Moreover, by Lemma 3.2 ,

$$
\#\left(E_{1} \cap\left(l, l^{\prime}\right)\right) \leq l^{\prime}-l+m-1, \quad \forall l<l^{\prime} \leq k_{0}+m .
$$

If $E_{2} \cap\left[\tilde{n}_{i}, k_{0}+m+2\right]$ is a sampling sequence for $\left.V_{m}\right|_{\left.\tilde{n}_{i}, k_{0}+m+2\right]}$ for all $i \leq 0$, then we have $\left.\left(f_{1}-f_{2}\right)\right|_{\left[\tilde{n}_{i}, k_{0}+m+2\right]}=0$ for all $i \leq 0$. Hence $f_{1}(x)-f_{2}(x)=0$ for $x \leq k_{0}+m+2$. Therefore, $f_{1}-f_{2}=0$, which is impossible. Hence there is some $i \leq 0$ such that $E_{2} \cap\left[\tilde{n}_{i}, k_{0}+m+2\right]$ is not a sampling sequence for $\left.V_{m}\right|_{\left[\tilde{n}_{i}, k_{0}+m+2\right]}$.

Observe that $E_{2} \cap\left[\tilde{n}_{i}, \tilde{n}_{i}^{\prime}\right]$ and $E_{2} \cap\left[k_{0}+m, k_{0}+m+2\right]$ are sampling sequences for $\left.V_{m}\right|_{\left[\tilde{n}_{i}, \tilde{n}_{i}^{\prime}\right]}$ and $\left.V_{m}\right|_{\left[k_{0}+m, k_{0}+m+2\right]}$, respectively. By (4.9), we have

$$
\#\left(E_{2} \cap\left(\tilde{n}_{i}^{\prime}, k_{0}+m\right)\right) \geq 2\left(k_{0}+m-\tilde{n}_{i}^{\prime}\right)-1-\left(k_{0}+m-\tilde{n}_{i}^{\prime}+m-1\right)
$$




$$
=k_{0}-\tilde{n}_{i}^{\prime}
$$

Hence

$$
\#\left(E_{2} \cap\left[\tilde{n}_{i}, k_{0}+m+2\right]\right) \geq k_{0}-\tilde{n}_{i}+2 m+2 .
$$

By Proposition 2.6, there exist integers $l<l^{\prime}$ such that $\tilde{n}_{i} \leq l<l^{\prime} \leq k_{0}+m$ and $\#\left(E_{2} \cap\left(l, l^{\prime}\right)\right) \leq l^{\prime}-l-m-1$. Hence \# $\left(E_{1} \cap\left(l, l^{\prime}\right)\right) \geq l^{\prime}-l+m$, which contradicts with (4.9).

It follows that there exist some $l<l^{\prime} \leq k_{0}+m$ such that $E_{1} \cap\left[l, l^{\prime}\right]$ is a sampling sequence for $\left.V_{m}\right|_{\left[l, l^{\prime}\right]}$. Since $E_{2} \cap\left[k_{0}+m, k_{0}+m+2\right]$ is a sampling sequence for $\left.V_{m}\right|_{\left[k_{0}+m, k_{0}+m+2\right]}$, we see from the proof of Lemma 3.4 that there exist integers $i_{1}<i_{1}^{\prime}=i_{2}<i_{2}^{\prime}$ such that $i_{2} \leq k_{0}+m$ and $E_{l} \cap\left[i_{l}, i_{l}^{\prime}\right]$ is a sampling sequence for $\left.V_{m}\right|_{\left[i_{l}, i_{l}^{\prime}\right]}, l=1,2$. Hence

$$
c_{i} \pm c_{i}^{\prime}=0, \quad i_{2}-m \leq i \leq i_{2}-1 .
$$

Since $c_{k_{0}}+c_{k_{0}}^{\prime} \neq 0$, we have $i_{2} \leq k_{0}$. If there is some $k_{1}<i_{2}-m$ such that

$$
c_{k_{1}}+c_{k_{1}}^{\prime} \neq 0 \quad \text { or } \quad c_{k_{1}}-c_{k_{1}}^{\prime} \neq 0
$$

then both $f_{1}$ and $f_{2}$ are separable.

In fact, we see from (P1) and (P2) that $E \cap\left[i, i_{2}\right]$ is a sampling sequence for $\left.V_{m}\right|_{\left[i, i_{2}\right]}$ provided $i_{2}-i$ is large enough. Now we see from (4.10) that $\left.f_{l}\right|_{\left[i, i_{2}\right]} \neq 0, l=1$, 2. Similarly we can show that $\left.f_{l}\right|_{\left[i_{2}, k_{0}+m+2\right]} \neq 0, l=1,2$. Hence both $f_{1}$ and $f_{2}$ are separable.

It remains to consider the case of $c_{n} \pm c_{n}^{\prime}=0$ for all $n \leq i_{2}-1$. In this case, we have

$$
\begin{aligned}
f_{1}+f_{2} & =\sum_{i_{2} \leq n \leq k_{0}} c_{n} \varphi_{m}(\cdot-k), \\
f_{1}-f_{2} & =\sum_{i_{2} \leq n \leq k_{0}} c_{n}^{\prime} \varphi_{m}(\cdot-k),
\end{aligned}
$$

$\left.\left(f_{1}+f_{2}\right)\right|_{E_{1}}=0$ and $\left.\left(f_{1}-f_{2}\right)\right|_{E_{2}}=0$.

Let $E_{0}=\left(E \cap\left[i_{2}, k_{0}+m+2\right]\right) \cup F$, where $F \subset\left(i_{2}-1, i_{2}\right) \cup\left(k_{0}+m+1, k_{0}+m+2\right)$, $\#\left(F \cap\left(i_{2}-1, i_{2}\right)\right)=\#\left(F \cap\left(k_{0}+m+1, k_{0}+m+2\right)\right)=m+3$. By Theorem 1.2, $E_{0}$ is a phaseless sampling sequence for $\left.V_{m}\right|_{\left[i_{2}-1, k_{0}+m+2\right]}$. Since $\left|f_{1}(x)\right|=\left|f_{2}(x)\right|$ for $x \in E_{0}$ and $f_{1} \pm f_{2} \neq 0$, both $f_{1}$ and $f_{2}$ are separable. This completes the proof for $m \geq 2$.

Next we consider the case of $m=1$. If $k_{1}=-\infty$ and $k_{2}=\infty$, then the above arguments also work for $m=1$.

If $k_{2}<\infty$, then $E \cap\left[n_{k_{2}}-1, n\right]$ is a phaseless sampling sequence for $\left.V_{1}\right|_{\left[n_{k_{2}}-1, n\right]}$ for any $n>n_{k_{2}}$, thanks to Theorem 1.2, If $f_{1}$ or $f_{2}$ is nonseparable, then

$$
\left.\left(f_{1}+f_{2}\right)\right|_{\left[n_{k_{2}}-1, n\right]}=0 \quad \text { or }\left.\quad\left(f_{1}-f_{2}\right)\right|_{\left[n_{k_{2}}-1, n\right]}=0, \quad n>n_{k_{2}} .
$$

We conclude that one of $f_{1}+f_{2}$ and $f_{1}-f_{2}$ must be zero on $\left[n_{k_{2}}-1, \infty\right)$. In fact, if $\left.\left(f_{1}+f_{2}\right)\right|_{\left[n_{k_{2}}-1, n\right]} \neq 0$ for some $n>n_{k_{2}}$, then $\left.\left(f_{1}-f_{2}\right)\right|_{\left[n_{k_{2}}-1, n\right]}=0$ for all $n>n_{k_{2}}$.

Suppose that $f_{1}=f_{2}$ on $\left[n_{k_{2}}-1, \infty\right)$. Let $\tilde{E}_{2}=E_{2} \cup\left\{n+x: n \geq n_{k_{2}}, x=1 / 3,2 / 3,1\right\}$ and $\tilde{E}=E_{1} \cup \tilde{E}_{2}$. Then $\left.\left(f_{1}-f_{2}\right)\right|_{\tilde{E}_{2}}=0$ and $\#(\tilde{E} \cap[n, n+1]) \geq 3$ for $n \geq n_{k_{2}}$. 
For the case of $k_{1}>-\infty$, with similar arguments we get $\tilde{E}=\tilde{E}_{1} \cup \tilde{E}_{2}$ such that $\left.\left(f_{1}+f_{2}\right)\right|_{\tilde{E}_{1}}=0,\left.\left(f_{1}-f_{2}\right)\right|_{\tilde{E}_{2}}=0$, and $\#(\tilde{E} \cap[n, n+1]) \geq 3$ for $n \leq n_{k_{1}}-1$. Now we see from previous arguments that both $f_{1}$ and $f_{2}$ are separable.

Finally, suppose that $\left|f_{1}(x)\right|=\left|f_{2}(x)\right|$ on $E$ for some $f_{1}, f_{2} \in V_{m}$ and $E$ meets (P1) and (P2) for $m \geq 2$ or (P1) and (P2') for $m=1$. We see from the above arguments that there exist integers $i_{k}<i_{k}^{\prime} \leq i_{k+1}<i_{k+1}^{\prime}, k \in \mathbb{Z}$ such that $\left|f_{1}(x)\right|=\left|f_{2}(x)\right|$ on $\left[i_{k}, i_{k}^{\prime}\right]$, $k \in \mathbb{Z}$. By Theorem 1.2 , it is easy to see that $\left|f_{1}(x)\right|=\left|f_{2}(x)\right|$ for any $x \in \mathbb{R}$. This completes the proof.

\section{$5 \quad$ Examples}

In this section, we give some examples to illustrate our main results on the characterization of phaseless sampling sequences. The first one is on the almost phaseless sampling.

Example 5.1 Let $N_{1}, N_{2}$ and $K$ be integers such that $N_{1}<N_{2}$. Define

$$
x_{i}=N_{1}+\frac{i-1}{K-1}\left(N_{2}-N_{1}\right), \quad 1 \leq i \leq K .
$$

It follows from Theorem 2.2 that $E=\left\{x_{i}: 1 \leq i \leq K\right\}$ is an almost phaseless sampling sequence for $\left.V_{m}\right|_{\left[N_{1}, N_{2}\right]}$ if $K \geq N_{2}-N_{1}+m+1$.

The second example is on the local phaseless sampling.

Example 5.2 Let $N_{1}, N_{2}$ and $K$ be integers such that $N_{1}<N_{2}-2$. Define

$$
x_{i}=N_{1}+1+\frac{i-1}{K-1}\left(N_{2}-N_{1}-2\right), \quad 1 \leq i \leq K,
$$

and

$$
y_{i}=N_{1}+\frac{i}{m+1}, \quad z_{i}=N_{2}-\frac{i}{m+1}, \quad 0 \leq i \leq m .
$$

We see from Theorem 1.2 that $E=\left\{x_{i}: 1 \leq i \leq K\right\} \cup\left\{y_{i}: 0 \leq i \leq m\right\} \cup\left\{z_{i}: 0 \leq i \leq m\right\}$ is a phaseless sampling sequence for $\left.V_{m}\right|_{\left[N_{1}, N_{2}\right]}$ if $K \geq 2\left(N_{2}-N_{1}\right)-3$.

The third example is on the global phaseless sampling, which is a simple consequence of Theorem 1.3. It illustrates the difference between the case of $m=1$ and the other case of $m>1$.

Example 5.3 Let $\alpha>\beta \geq 0$ be constants.

(i). For $m \geq 2,\{n \alpha+\beta: n \in \mathbb{Z}\}$ is a phaseless sampling sequence for $V_{m}$ if and only if $0<\alpha<1 / 2$.

(ii). For $m=1,\{n \alpha+\beta: n \in \mathbb{Z}\}$ is a phaseless sampling sequence for $V_{m}$ if and only if $0<\alpha<1 / 2$ or $\alpha=1 / 2$ and $\beta=0$. 


\section{References}

[1] R. Alaifari, I. Daubechies, P. Grohs, and G. Thakur. Reconstructing real-valued functions from unsigned coefficients with respect to wavelet and other frames. Journal of Fourier Analysis and Applications, pages 1-15, 2016.

[2] R. Alaifari, I. Daubechies, P. Grohs, and R. Yin. Stable phase retrieval in infinite dimensions. arXiv preprint arXiv:1609.00034, 2016.

[3] S. Bahmanpour, J. Cahill, P. G. Casazza, J. Jasper, and L. M. Woodland. Phase retrieval and norm retrieval. arXiv preprint arXiv:1409.8266, 2014.

[4] R. Balan, P. Casazza, and D. Edidin. On signal reconstruction without phase. Appl. Comput. Harmon. Anal., 20(3):345-356, 2006.

[5] A. S. Bandeira, J. Cahill, D. G. Mixon, and A. A. Nelson. Saving phase: Injectivity and stability for phase retrieval. Applied and Computational Harmonic Analysis, 37(1):106 - 125, 2014.

[6] A. S. Bandeira and Y. Chen. Phase retrieval from power spectra of masked signals. Information and Inference: A Journal of the IMA, 3:83-102, 2014.

[7] B. G. Bodmann and N. Hammen. Algorithms and error bounds for noisy phase retrieval with low-redundancy frames. Applied and Computational Harmonic Analysis, pages -, 2016.

[8] J. Cahill, P. G. Casazza, and I. Daubechies. Phase retrieval in infinite-dimensional Hilbert spaces. Trans. Amer. Math. Soc. Ser. B, 3:63-76, 2016.

[9] J. Cahill, P. G. Casazza, J. Jasper, and L. M. Woodland. Phase retrieval. arXiv preprint arXiv:1508.06687, 2015.

[10] J. Cahill, P. G. Casazza, J. Peterson, and L. Woodland. Phase retrieval by projections. Houston J. Math., 42(2):537-558, 2016.

[11] P. G. Casazza, D. Ghoreishi, S. Jose, and J. C. Tremain. Norm retrieval and phase retrieval by projections. Axioms, 6(1), 2017.

[12] H. Chang, Y. Lou, M. K. Ng, and T. Zeng. Phase retrieval from incomplete magnitude information via total variation regularization. SIAM J. Sci. Comput., 38(6):A3672A3695, 2016.

[13] Y. Chen, C. Cheng, Q. Sun, and H. Wang. Phase retrieval of real-valued signals in a shift-invariant space. CoRR, abs/1603.01592, 2016.

[14] C. Cheng, J. Jiang, and Q. Sun. Phaseless sampling and reconstruction of real-valued signals in shift-invariant spaces. arXiv preprint arXiv:1702.06443, 2017. 
[15] D. Edidin. Projections and phase retrieval. Appl. Comput. Harmon. Anal., 42(2):350$359,2017$.

[16] M. Fickus, D. G. Mixon, A. A. Nelson, and Y. Wang. Phase retrieval from very few measurements. Linear Algebra Appl., 449:475-499, 2014.

[17] D. Gross, F. Krahmer, and R. Kueng. Improved recovery guarantees for phase retrieval from coded diffraction patterns. Appl. Comput. Harmon. Anal., 42(1):37-64, 2017.

[18] K. Huang, Y. C. Eldar, and N. D. Sidiropoulos. Phase retrieval from 1D Fourier measurements: convexity, uniqueness, and algorithms. IEEE Trans. Signal Process., 64(23):6105-6117, 2016.

[19] M. Iwen, A. Viswanathan, and Y. Wang. Robust sparse phase retrieval made easy. Appl. Comput. Harmon. Anal., 42(1):135-142, 2017.

[20] M. A. Iwen, A. Viswanathan, and Y. Wang. Fast phase retrieval from local correlation measurements. SIAM J. Imaging Sci., 9(4):1655-1688, 2016.

[21] S. Mallat and I. Waldspurger. Phase retrieval for the cauchy wavelet transform. Journal of Fourier Analysis and Applications, 21(6):1251-1309, 2015.

[22] V. Pohl, F. Yang, and H. Boche. Phaseless signal recovery in infinite dimensional spaces using structured modulations. Journal of Fourier Analysis and Applications, 20(6):1212-1233, 2014.

[23] V. Pohl, F. Yang, and H. Boche. Phase retrieval from low-rate samples. Sampling Theory in Signal and Image Processing, 14(1):71-99, 2015.

[24] C. Qian, N. D. Sidiropoulos, K. Huang, L. Huang, and H. C. So. Phase retrieval using feasible point pursuit: algorithms and Cramér-Rao bound. IEEE Trans. Signal Process., 64(20):5282-5296, 2016.

[25] T. Qiu, P. Babu, and D. P. Palomar. PRIME: phase retrieval via majorizationminimization. IEEE Trans. Signal Process., 64(19):5174-5186, 2016.

[26] L. L. Schumaker. Spline functions: basic theory. John Wiley \& Sons, Inc., New York, 1981. Pure and Applied Mathematics, A Wiley-Interscience Publication.

[27] Y. Shechtman, Y. C. Eldar, O. Cohen, H. N. Chapman, J. Miao, and M. Segev. Phase retrieval with application to optical imaging: A contemporary overview. IEEE Signal Processing Magazine, 32(3):87-109, May 2015.

[28] B. A. Shenoy, S. Mulleti, and C. S. Seelamantula. Exact phase retrieval in principal shift-invariant spaces. IEEE Transactions on Signal Processing, 64(2):406-416, Jan 2016 . 
[29] W. Sun and X. Zhou. Characterization of local sampling sequences for spline subspaces. Adv. Comput. Math., 30(2):153-175, 2009.

[30] G. Thakur. Reconstruction of bandlimited functions fromunsigned samples. Journal of Fourier Analysis and Applications, 17(4):720-732, 2011.

[31] A. M. Tillmann, Y. C. Eldar, and J. Mairal. DOLPHIn - dictionary learning for phase retrieval. IEEE Trans. Signal Process., 64(24):6485-6500, 2016.

[32] F. Yang, V. Pohl, and H. Boche. Phase retrieval via structured modulations in paleywiener spaces. arXiv preprint arXiv:1302.4258, 2013.

[33] W. Zhong. Almost phase retrieval with few intensity measurements. Mathematical Problems in Engineering, 2014, 2014. 9 pages. 